\title{
Approximation and support theorem for a wave equation in two space dimensions
}

\author{
ANNIE MILLET ${ }^{1}$ and MARTA SANZ-SOLÉ 2 \\ ${ }^{1}$ Laboratoire de Probabilités, Université Paris VI, 4 place Jussieu, 75252 Paris Cedex 05, \\ France.E-mail: amil@ccr.jussieu.fr \\ ${ }^{2}$ Facultat de Matemàtiques, Universitat de Barcelona, Gran Via 585, 08007 Barcelona Spain. E- \\ mail:sanz@mat.ub.es
}

We prove a characterization of the support of the law of the solution for a stochastic wave equation with two-dimensional space variable, driven by a noise white in time and correlated in space. The result is a consequence of an approximation theorem, in the convergence of probability, for equations obtained by smoothing the random noise. For some particular classes of coefficients, approximation in the $L^{p}$-norm for $p \geqslant 1$ is also proved.

Keywords: approximations; stochastic partial differential equations; support theorem

\section{Introduction and preliminaries}

In this paper we characterize the topological support of the law of the solution to the stochastic wave equation with two-dimensional spatial variable

$\left(\frac{\partial^{2}}{\partial t^{2}}-\Delta\right) u(t, x)=\sigma(u(t, x)) F(\mathrm{~d} t, \mathrm{~d} x)+b(u(t, x)), \quad u(0, x)=u_{0}(x), \quad \frac{\partial u}{\partial t}(0, x)=v_{0}(x)$

where $(t, x) \in\left[0, \infty\left[\times \mathbb{R}^{2}\right.\right.$. In this equation $F(t, x)$ is a generalized Gaussian field with covariance

$$
\mathrm{E}(F(t, x) F(s, y))=\delta(t-s) f(|x-y|),
$$

where $\delta$ denotes the Dirac delta function and $f$ is a continuous function on $] 0, \infty[$ satisfying an integrability condition made precise in assumption $(\mathrm{C} 1)$ below. In addition, the functional $J: \mathscr{D}\left(\mathbb{R}^{3}\right) \times \mathscr{D}\left(\mathbb{R}^{3}\right) \rightarrow \mathbb{R}$ given by

$$
J(\varphi, \psi)=\int_{0}^{\infty} \mathrm{d} t \int_{\mathbb{R}^{2}} \mathrm{~d} x \int_{\mathbb{R}^{2}} \mathrm{~d} y \varphi(t, x) f(|x-y|) \psi(t, y)
$$

is positive definite. With these hypotheses the process $\left\{F(t, x),(t, x) \in\left[0, \infty\left[\times \mathbb{R}^{2}\right\}\right.\right.$ exists.

We consider the mild formulation of (1.1). Let

$$
S(t, x)=\frac{1}{2 \pi}\left(t^{2}-|x|^{2}\right)^{-1 / 2} 1_{\{|x|<t\}} ;
$$


then a solution of (1.1) is a stochastic process $\left\{u(t, x),(t, x) \in\left[0, \infty\left[\times \mathbb{R}^{2}\right\}\right.\right.$ satisfying

$$
\begin{aligned}
u(t, x)= & \int_{\mathbb{R}^{2}} S(t, x-y) v_{0}(y) \mathrm{d} y+\frac{\partial}{\partial t}\left(\int_{\mathbb{R}^{2}} S(t, x-y) u_{0}(y) \mathrm{d} y\right) \\
& +\int_{0}^{t} \int_{\mathbb{R}^{2}} S(t-s, x-y)[\sigma(u(s, y)) F(\mathrm{~d} s, \mathrm{~d} y)+b(u(s, y)) \mathrm{d} s \mathrm{~d} y] .
\end{aligned}
$$

Consider the following set of assumptions on the elements defining (1.3):

(C1) There exist $\beta \in(0,1), r_{0}>0$, such that, for $0<t<r_{0}$,

$$
\int_{0}^{t} r f(r) \ln \left(1+\frac{t}{r}\right) \mathrm{d} r \leqslant C t^{\beta}
$$

(C2) $u_{0}: \mathbb{R}^{2} \rightarrow \mathbb{R}$ is of class $\mathscr{C}^{1}$ and bounded with $\beta / 2(1+\beta)$-Hölder continuous partial derivatives, and $v_{0}: \mathbb{R}^{2} \rightarrow \mathbb{R}$ is such that $\left|v_{0}\right|+\left|\nabla u_{0}\right| \in L^{q_{0}}$ for some $\left.\left.q_{0} \in\right] 4, \infty\right]$.

(C3) $\sigma, b: \mathbb{R} \rightarrow \mathbb{R}$ are globally Lipschitz functions.

Equation (1.3) was solved by Dalang and Frangos (1998) up to some small time $t_{0}>0$. In a previous paper (Millet and Sanz-Solé 1999), we proved that, assuming (C1), (C2) and (C3), equation (1.3) has a unique solution on $[0, T] \times \mathbb{R}^{2}$ for any $T>0$. Moreover, the trajectories of $u$ are $\gamma$-Hölder continuous in $(t, x) \in[0, T] \times K$ for every $\gamma \in(0, \beta / 2(1+\beta))$ and every compact subset $K$ of $\mathbb{R}^{2}$.

Let $\mathscr{E}$ denote the inner product space of measurable functions $\varphi: \mathbb{R}^{2} \rightarrow \mathbb{R}$ such that

$$
\int_{\mathbb{R}^{2}} \mathrm{~d} x \int_{\mathbb{R}^{2}} \mathrm{~d} y|\varphi(x)| f(|x-y|)|\varphi(y)|<+\infty,
$$

endowed with the scalar product

$$
\langle\varphi, \psi\rangle_{\mathscr{E}}:=\int_{\mathbb{R}^{2}} \mathrm{~d} x \int_{\mathbb{R}^{2}} \mathrm{~d} y \varphi(x) f(|x-y|) \psi(y),
$$

and let $\tilde{H}$ denote the completion of $\mathscr{E}$. Set $H=L^{2}([0, T], \tilde{H})$; notice that $H$ and $\tilde{H}$ need not be spaces of functions. For $h \in H$, let $\left\{\Phi^{h}(t, x),(t, x) \in[0, \infty) \times \mathbb{R}^{2}\right\}$ be the solution of

$$
\begin{aligned}
\Phi^{h}(t, x)= & \int_{\mathbb{R}^{2}} S(t, x-y) v_{0}(y) \mathrm{d} y+\frac{\partial}{\partial t}\left(\int_{\mathbb{R}^{2}} S(t, x-y) u_{0}(y) \mathrm{d} y\right) \\
& +\left\langle S(t-\cdot, x-*) \sigma\left(\Phi^{h}(\cdot, *)\right), h\right\rangle_{H}+\int_{0}^{t} \int_{\mathbb{R}^{2}} S(t-s, x-y) b\left(\Phi^{h}(s, y)\right) \mathrm{d} s \mathrm{~d} y .
\end{aligned}
$$

Set

$$
\|\varphi\|_{\gamma, K}=\sup _{\substack{t \in[0, T] \\ x \in K}}|\varphi(t, x)|+\sup _{\substack{t, t^{\prime} \in[0, T] \\ x, x^{\prime} \in K \\ t \neq t^{\prime}, x \neq x^{\prime}}} \frac{\left|\varphi(t, x)-\varphi\left(t^{\prime}, x^{\prime}\right)\right|}{\left(\left|t-t^{\prime}\right|+\left|x-x^{\prime}\right|\right)^{\gamma}}
$$


We prove in Section 2 that the support of the law of $\{u(t, x),(t, x) \in[0, T] \times K\}$ is the closure with respect to the norm $\|\cdot\|_{\gamma, K}$ of the set of functions $\left\{\Phi^{h}, h \in H\right\}$, where $\left\{\Phi^{h}(t, x),(t, x) \in[0, T] \times K\right\}$ is the solution of (1.4). The proof is based on an approximation result for equations more general than (1.3) constructed by smoothing the random noise $F(t, x)$. We refer the reader to Millet and Sanz-Solé (1994a; 1994b) and Bally et al. (1995) for a presentation of the method and applications to stochastic differential and stochastic partial differential equations.

In the framework of stochastic partial differential equations, the regularization of the noise raises technical difficulties connected with the explosion of the corresponding integral (see, for instance, Bally et al. 1995). This problem does not appear here for the following reasons. The noise $F$ is smoother than space-time white noise. On the other hand, the integrability condition (C1) and Lemma A.1 in Millet and Sanz-Solé (1999) yield

$$
\mu(t) \leqslant C t^{\beta+1}
$$

(see (A.2) and (A.11) below). This fact prevents explosions, as is made explicit in the proofs.

We now introduce some preliminaries and notation used throughout this paper. Let $\left\{e_{j}, j \in \mathbb{N}\right\}$ be a sequence of functions of $\mathscr{E}$ which is a complete orthonormal system of $\tilde{H}$ and is taken to be fixed. Define

$$
W_{j}(t)=\int_{0}^{t} \int_{\mathbb{R}^{2}} e_{j}(x) F(\mathrm{~d} s, \mathrm{~d} x), j \in \mathbb{N}, t \in[0, T] .
$$

Clearly $\left\{W_{j}, j \in \mathbb{N}\right\}$ is a sequence of independent Brownian motions.

Let $\mathscr{H}$ be the separable Hilbert space of functions $k:[0, T] \rightarrow \mathbb{R}^{\mathbb{N}}$ such that $\int_{0}^{T} \sum_{j=1}^{\infty}\left|k_{j}(s)\right|^{2} \mathrm{~d} s<\infty$ and endowed with the inner product

$$
\langle k, \bar{k}\rangle_{\mathscr{C}}=\int_{0}^{T} \sum_{j=1}^{\infty} k_{j}(s) \bar{k}_{j}(s) \mathrm{d} s
$$

Notice that the mapping

$$
\begin{aligned}
\mathscr{J}: H & \rightarrow \mathscr{H} \\
\varphi & \mapsto\left(\left\langle\varphi(s, *), e_{j}(*)\right\rangle_{\tilde{H}}\right)_{j \in \mathbb{N}}
\end{aligned}
$$

provides an isometry between $H$ and $\mathscr{H}$.

Let $\mathscr{F}_{t}=\sigma\left(F([0, s] \times A) ; \quad 0 \leqslant s \leqslant t, \quad A \in \mathscr{B}\left(\mathbb{R}^{2}\right)\right), \quad t \geqslant 0$. For any $\mathscr{F}_{t}$-predictable process $\varphi \in L^{2}(\Omega ; H)$ we have

$$
\int_{0}^{t} \int_{\mathbb{R}^{2}} \varphi(s, x) F(\mathrm{~d} s, \mathrm{~d} x)=\sum_{j=1}^{\infty} \int_{0}^{t}\left\langle\varphi(s, *), e_{j}(*)\right\rangle_{\tilde{H}} W_{j}(\mathrm{~d} s),
$$

$t \in[0, T]$, so that the stochastic integral with respect to the martingale measure $F$ can also be viewed as a stochastic integral with respect to the infinite-dimensional Gaussian process $\left\{W_{j}(t), t \in[0, T], j \in \mathbb{N}\right\}$. We introduce smooth approximations of $F$ constructed as follows. Fix $n \in \mathbb{N}$ and let $\mathscr{P}_{n}$ be the partition of $[0, T]$ determined by $i T / 2^{n}, i=0,1, \ldots, 2^{n}$. 
Denote by $\Delta_{i}$ the interval $\left[i T / 2^{n},(i+1) T / 2^{n}\right)$ and by $\left|\Delta_{i}\right|$ its length. We write $W_{j}\left(\Delta_{i}\right)$ for the increment $W_{j}\left((i+1) T / 2^{n}\right)-W_{j}\left(i T / 2^{n}\right), i=0, \ldots, 2^{n}-1$. Define

$$
W^{n}=\left(W_{j}^{n}=\int_{0} \dot{W}_{j}^{n}(s) \mathrm{d} s, j \in \mathbb{N}\right),
$$

where, for $j>n$,

$$
\dot{W}_{j}^{n}=0,
$$

and, for $1 \leqslant j \leqslant n$,

$$
\dot{W}_{j}^{n}(t)= \begin{cases}\sum_{i=1}^{2^{n}} 2^{n} T^{-1} W_{j}\left(\Delta_{i-1}\right) 1_{\Delta_{i}}(t) & \text { if } t \in\left[2^{-n} T, T\right], \\ 0 & \text { if } t \in\left[0,2^{-n} T\right) .\end{cases}
$$

Set

$$
\omega^{n}(t, x)=\sum_{j \in \mathbb{N}} \dot{W}_{j}^{n}(t) e_{j}(x) .
$$

It is easy to check that almost surely, $\omega^{n} \in H$ and, more precisely,

$$
\left\|\omega^{n}\right\|_{L^{p}(\Omega ; H)} \leqslant C n^{1 / 2} 2^{n / 2}, \quad \forall p \in[1, \infty) .
$$

Indeed, fix $p \in[2, \infty)$; then

$$
\begin{aligned}
\left\|\omega^{n}\right\|_{L^{p}(\Omega ; H)}^{p} & =\mathrm{E}\left(\left|\sum_{j=1}^{n} \sum_{i=1}^{2^{n}} 2^{2 n} T^{-2}\right| \Delta_{i}\left|W_{j}\left(\Delta_{i-1}\right)^{2}\right|^{p / 2}\right) \\
& \leqslant C n^{p / 2} 2^{n p / 2} .
\end{aligned}
$$

Moreover, for any $0 \leqslant t_{1} \leqslant t_{2} \leqslant T$, similar computations imply

$$
\left\|\omega^{n} 1_{\left[t_{1}, t_{2}\right]}\right\|_{L^{p}(\Omega ; H)} \leqslant C n^{1 / 2} 2^{n / 2}\left|t_{2}-t_{1}\right|^{1 / 2} .
$$

Let $(\bar{\Omega}, \overline{\mathscr{F}}, \bar{P})$ be the canonical space associated with a standard Brownian motion. Denote by $(\Omega, \mathscr{F}, P)$ the product space $\left(\bar{\Omega}^{\mathbb{N}}, \overline{\mathscr{F}}^{\otimes \mathbb{N}}, \bar{P}^{\otimes \mathbb{N}}\right)$, which will be our reference probability space.

Set $\bar{k}(t)=\int_{0}^{t} k(s) \mathrm{d} s$ for $k \in \mathscr{H}$. For any integer $n \geqslant 1$, let $T_{n}^{k}$ denote the transformation of $\Omega$ defined by

$$
T_{n}^{k}(\omega)=\omega+\bar{k}-W^{n}(\omega) .
$$

Notice that $T_{n}^{k}(\omega)=\omega+\int_{0}^{*} \varphi_{n}(s, \omega) \mathrm{d} s$, where $\left\{\varphi_{n}(t, \omega), t \in[0, T]\right\}$ is an $\mathscr{H}$-valued process adapted to the filtration generated by $\left\{W_{j}(t), t \in[0,1], j \in \mathbb{N}\right\}$. Therefore, by Girsanov's theorem, $P_{\circ}\left(T_{n}^{k}\right)^{-1} \ll P$. This fact will be used in the proof of Theorem 2.1.

The paper is organized as follows. In Section 2 we prove the characterization of the support by means of an approximation in probability. In Section 3 we prove approximations 
in $L^{p}$-norm under stronger hypotheses on the coefficients. As usual, all constants are denoted by $C$, regardless of their values.

\section{Approximation in probability and support theorem}

The purpose of this section is to prove the following result:

Theorem 2.1. Assume (C1)-(C3), fix a compact set $K \subset \mathbb{R}^{2}$ and let $\{u(t, x), t \in[0, T]$, $x \in K\}$ be the solution of (1.3). Then for any $\gamma \in(0, \beta / 2(1+\beta))$ the topological support of the law of $u$ in the space $\mathscr{C}^{\gamma}([0, T] \times K)$ of $\gamma$-Hölder continuous functions in $(t, x)$ is given by the closure in $\mathscr{C}^{\gamma}([0, T] \times K)$ of the set of functions $\left\{\Phi^{h}, h \in H\right\}$, where $\left\{\Phi^{h}(t, x), t \in[0, T], x \in K\right\}$ is the solution of (1.4).

The proof of Theorem 2.1 is a consequence of an approximation result, concerning convergence in probability, for an equation more general than (1.3).

More precisely, let us introduce the following hypothesis:

(C3') The coefficients $A, B, D, b: \mathbb{R} \rightarrow \mathbb{R}$ are globally Lipschitz functions.

Then we consider the evolution equations

$$
\begin{aligned}
& X_{n}(t, x)= X^{0}(t, x)+\int_{0}^{t} \int_{\mathbb{R}^{2}} S(t-s, x-y) A\left(X_{n}(s, y)\right) F(\mathrm{~d} s, \mathrm{~d} y) \\
&+\left\langle S(t-\cdot, x-*) B\left(X_{n}(\cdot, *)\right), \omega^{n}\right\rangle_{H}+\left\langle S(t-\cdot, x-*) D\left(X_{n}(\cdot, *)\right), h\right\rangle_{H} \\
& \quad+\int_{0}^{t} \int_{\mathbb{R}^{2}} S(t-s, x-y) b\left(X_{n}(s, y)\right) \mathrm{d} s \mathrm{~d} y, \\
& X(t, x)=X^{0}(t, x)+\int_{0}^{t} \int_{\mathbb{R}^{2}} S(t-s, x-y)(A+B)(X(s, y)) F(\mathrm{~d} s, \mathrm{~d} y) \\
&+\langle S(t-\cdot, x-*) D(X(\cdot, *)), h\rangle_{H}+\int_{0}^{t} \int_{\mathbb{R}^{2}} S(t-s, x-y) b(X(s, y)) \mathrm{d} s \mathrm{~d} y,
\end{aligned}
$$

where $n \geqslant 1, A, B, D, b$ satisfy $\left(\mathrm{C}^{\prime}\right), h \in H, \omega^{n}$ is defined in (1.11) and

$$
X^{0}(t, x)=\int_{\mathbb{R}^{2}} S(t, x-y) v_{0}(y) \mathrm{d} y+\frac{\partial}{\partial t}\left(\int_{\mathbb{R}^{2}} S(t, x-y) u_{0}(y) \mathrm{d} y\right) .
$$

Our aim is to prove the following:

Proposition 2.1. Assume (C1), (C2) and (C3'). For any $\gamma \in(0, \beta / 2(1+\beta)), \eta>0$ and every compact set $K \subset \mathbb{R}^{2}$, 


$$
\lim _{n \rightarrow \infty} P\left(\left\|X_{n}-X\right\|_{\gamma, K}>\eta\right)=0,
$$

where $\|\cdot\|_{\gamma, K}$ has been defined in (1.5).

We first show that Theorem 2.1 is an easy consequence of this proposition.

Proof of Theorem 2.1. Assume that Proposition 2.1 has been proved. For $n \geqslant 1$, set

$$
\begin{gathered}
u_{n}(t, x)=X^{0}(t, x)+\left\langle S(t-\cdot, x-*) \sigma\left(u_{n}(\cdot, *)\right), \omega^{n}\right\rangle_{H} \\
\quad+\int_{0}^{t} \int_{\mathbb{R}^{2}} S(t-s, x-y) b\left(u_{n}(s, y)\right) \mathrm{d} s \mathrm{~d} y, \\
v_{n}(t, x)=X^{0}(t, x)+\int_{0}^{t} \int_{\mathbb{R}^{2}} S(t-s, x-y) \sigma\left(v_{n}(s, y)\right) F(\mathrm{~d} s, \mathrm{~d} y) \\
+\left\langle S(t-\cdot, x-*) \sigma\left(v_{n}(\cdot, *)\right), h-\omega^{n}\right\rangle_{H} \\
+\int_{0}^{t} \int_{\mathbb{R}^{2}} S(t-s, x-y) b\left(v_{n}(s, y)\right) \mathrm{d} s \mathrm{~d} y .
\end{gathered}
$$

Clearly, equations (2.5) and (2.6) are particular cases of (2.1), while equations (1.3) and (1.4) are particular cases of (2.2), obtained by choosing $A=D=0, B=\sigma$ and $A=D=\sigma$, $B=-\sigma$, respectively.

Moreover, $u_{n}=\Phi^{\omega^{n}}$. Given $h \in H$, set $k=\mathscr{J}(h)$, where $\mathscr{J}$ is the isometry defined in (1.7). Then, by (1.8), equation (2.6) can be rewritten as follows:

$$
\begin{aligned}
v_{n}(t, x)= & X^{0}(t, x)+\sum_{j=1}^{\infty} \int_{0}^{t}\left\langle S(t-s, x-*) \sigma\left(v_{n}(s, *)\right), e_{j}\right\rangle_{\tilde{H}} W_{j}(\mathrm{~d} s) \\
& +\sum_{j=1}^{\infty} \int_{0}^{t}\left\langle S(t-s, x-*) \sigma\left(v_{n}(s, *)\right), e_{j}\right\rangle_{\tilde{H}}\left(k_{j}(s)-\dot{W}_{j}^{n}(s)\right) \mathrm{d} s \\
& +\int_{0}^{t} \int_{\mathbb{R}^{2}} S(t-s, x-y) b\left(v_{n}(s, y)\right) \mathrm{d} s \mathrm{~d} y,
\end{aligned}
$$

with $\dot{W}_{j}^{n}$ defined in (1.10). Therefore, $v^{n}(\omega)=u \circ T_{n}^{k}(\omega)$, where $T_{n}^{k}$ is the absolutely continuous transformation on $\Omega$ defined by (1.14).

The convergence (2.4) implies, for any $\eta>0$,

$$
\begin{array}{r}
\lim _{n \rightarrow \infty} P\left(\left\|\Phi^{\omega^{n}}-u\right\|_{\gamma, K}>\eta\right)=0, \\
\lim _{n \rightarrow \infty} P\left(\left\|u\left(T_{n}^{h}\right)-\Phi^{h}\right\|_{\gamma, K}>\eta\right)=0 .
\end{array}
$$

These two convergences yield the characterization of the support stated in Theorem 2.1 (see, for instance, Millet and Sanz-Solé (1994a; or 1994b). Indeed, since $\omega_{n} \in H$, the first convergence implies that the support of $u$ in $\mathscr{C}^{\gamma}([0, T] \times K)$ is included in the closure of 
$\left\{\Phi^{h}: h \in H\right\}$. Since $\omega^{n}$ is adapted, $P \circ\left(T_{n}^{h}\right)^{-1} \ll P$; the second convergence yields the converse inclusion.

The question of the existence and uniqueness of a solution to equations (2.1) and (2.2) is solved in Lemma A.1, which is a slight extension of Theorem 1.2 in Millet and Sanz-Solé (1999). We remark that the existence of a solution $X_{n}$ to (2.1) as well as upper estimates of $X_{n}$ require some localization, due to the term involving $\omega^{n}$ which has an unbounded $H$ norm. For this reason we localize $\omega^{n}$ as follows: for any positive integer $n, M \in \mathbb{N}_{+}$and $t \in[0, T]$, set

$$
A_{n, M}(t)=\left\{\sup _{1 \leqslant j \leqslant n} \sup _{1 \leqslant i \leqslant\left(\left[2^{n} t T^{-1}\right]-1\right)^{+}} 2^{n}\left|W_{j}\left(\Delta_{i}\right)\right| \leqslant M\right\}
$$

and

$$
\omega^{n, M}(t, x)=\omega^{n}(t, x) 1_{A_{n, M}(t)}
$$

Notice that

$$
\sup _{0 \leqslant t \leqslant T}\left\|\omega^{n, M}(t, *)\right\|_{\tilde{H}} \leqslant M \sqrt{n} .
$$

Fix $\alpha>(2 \ln 2)^{1 / 2}$ and, for every $n>0$, set

$$
M(n)=\alpha 2^{n / 2} n^{1 / 2}
$$

and

$$
A_{n}(t)=A_{n, M(n)}(t) .
$$

Lemma 2.1. The following convergence holds:

$$
\lim _{n \rightarrow \infty} P\left(A_{n}(T)^{c}\right)=0 .
$$

Proof. Let $Z$ denote an $N(0,1)$ random variable. Then

$$
\begin{aligned}
P\left(A_{n}(T)^{c}\right) & \leqslant n 2^{n} P\left(|Z|>2^{-n / 2} M(n)\right) \\
& \leqslant C n 2^{n} \frac{2^{n / 2}}{M(n)} \exp \left(-\frac{2^{-n} M(n)^{2}}{2}\right) \\
& =C \sqrt{n} \exp \left(-n\left(\frac{\alpha^{2}}{2}-\ln 2\right)\right) \underset{n \rightarrow \infty}{\rightarrow} 0 .
\end{aligned}
$$

Remark 2.1. Due to (2.8), on the set $A_{n}(T)$ we have

$$
\left\|\omega^{n}\right\|_{H} \leqslant C n 2^{n / 2}
$$

and, for any $0 \leqslant t \leqslant t^{\prime} \leqslant T$, on $A_{n}\left(t^{\prime}\right)$ we have 


$$
\left\|\omega^{n} 1_{\left[t, t^{\prime}\right]}\right\|_{H} \leqslant C n 2^{n / 2}\left|t^{\prime}-t\right|^{1 / 2} .
$$

In particular, if $\left[t, t^{\prime}\right] \subset \Delta_{i}$ for some $i=0, \ldots, 2^{n}-1$, on $A_{n}\left(t^{\prime}\right)$ we have

$$
\left\|\omega^{n} 1_{\left[t, t^{\prime}\right]}\right\|_{H} \leqslant C n .
$$

Lemma A.1 yields the existence and uniqueness of the solution $X_{n}$ to (2.1) and Remark 2.2 shows that the trajectories of $X_{n}$ almost surely have $\gamma$-Hölder continuous trajectories for $\gamma<\beta / 2(1+\beta)$; since $X$ is a particular case of $X_{n}$, it also has $\gamma$-Hölder continuous trajectories.

Our next purpose is to check that the sequence of processes $Y_{n}(t, x):=X_{n}(t, x)-$ $X(t, x), n \geqslant 1$, satisfies the requirements of Lemma A.2. To this end, we introduce some notation and prove several lemmas. For any $n \geqslant 1, t \in[0, T]$, set

$$
\begin{aligned}
& \underline{t}_{n}= \max \left\{k 2^{-n} T ; k=1, \ldots, 2^{n}-1: k 2^{-n} T \leqslant t\right\}, \\
& t_{n}= \max \left\{\underline{t}_{n}-2^{-n} T, 0\right\}, \\
& X_{n}^{-}(t, x)= X^{0}(t, x)+\int_{0}^{t_{n}} \int_{\mathbb{R}^{2}} S(t-s, x-y) A\left(X_{n}(s, y)\right) F(\mathrm{~d} s, \mathrm{~d} y) \\
&+\left\langle S(t-\cdot, x-*) B\left(X_{n}(\cdot, *)\right) 1_{\left[0, t_{n}\right]}(\cdot), \omega^{n}\right\rangle_{H} \\
&+\left\langle S(t-\cdot, x-*) D\left(X_{n}(\cdot, *)\right) 1_{\left[0, t_{n}\right]}(\cdot), h\right\rangle_{H} \\
&+\int_{0}^{t_{n}} \int_{\mathbb{R}^{2}} S(t-s, x-y) b\left(X_{n}(s, y)\right) \mathrm{d} s \mathrm{~d} y, \\
& X^{-}(t, x)=X^{0}(t, x)+\int_{0}^{t_{n}} \int_{\mathbb{R}^{2}} S(t-s, x-y)(A+B)(X(s, y)) F(\mathrm{~d} s, \mathrm{~d} y) \\
&+\left\langle S(t-\cdot, x-*) D(X(\cdot, *)) 1_{\left[0, t_{n}\right]}(\cdot), h\right\rangle_{H} \\
&+\int_{0}^{t_{n}} \int_{\mathbb{R}^{2}} S(t-s, x-y) b(X(s, y)) \mathrm{d} s \mathrm{~d} y .
\end{aligned}
$$

For convenience' sake, we do not write explicitly the fact that the process $X^{-}$depends on $n$. In what follows, \|\|$_{p}$ denotes the $L^{p}(\Omega)$-norm.

Lemma 2.2. Suppose that conditions (C1), (C2) and (C3') hold. Then, for any $p \in[1, \infty)$ and every integer $n \geqslant 1$,

$$
\sup _{(s, x) \in[0, T] \times \mathbb{R}^{2}}\left\|X(s, x)-X^{-}(s, x)\right\|_{p} \leqslant C 2^{-n(\beta+1) / 2}
$$

and 
Support theorem for a wave equation in two space dimensions

$$
\sup _{n \geqslant 1} \sup _{(s, x) \in[0, T] \times \mathbb{R}^{2}}\left\|X^{-}(s, x)\right\|_{p} \leqslant \infty .
$$

Proof. Set $\left\|X(t, x)-X^{-}(t, x)\right\|_{p}^{p} \leqslant C \sum_{i=1}^{3} V_{i}(t, x)$, with

$$
\begin{aligned}
& V_{1}(t, x)=\mathrm{E}\left(\left|\int_{t_{n}}^{t} \int_{\mathbb{R}^{2}} S(t-s, x-y)(A+B)(X(s, y)) F(\mathrm{~d} s, \mathrm{~d} y)\right|^{p}\right), \\
& V_{2}(t, x)=\mathrm{E}\left(\left.\left\langle S(t-\cdot, x-*) D(X(\cdot, *)) 1_{\left(t_{n}, t\right]}(\cdot), h\right\rangle_{H}\right|^{p}\right), \\
& V_{3}(t, x)=\mathrm{E}\left(\left|\int_{t_{n}}^{t} \int_{\mathbb{R}^{2}} S(t-s, x-y) b(X(s, y)) \mathrm{d} s \mathrm{~d} y\right|^{p}\right) .
\end{aligned}
$$

Burkholder's and Hölder's inequalities, along with (A.15) and (A.11), yield

$$
V_{1}(t, x) \leqslant C \mu\left(t-t_{n}\right)^{p / 2}\left(1+\sup _{(t, x) \in[0, T] \times \mathbb{R}^{2}} \mathrm{E}\left(|X(t, x)|^{p}\right)\right) \leqslant C 2^{-n(\beta+1) p / 2},
$$

with $\mu\left(t-t_{n}\right)$ given by (A.2).

The Cauchy-Schwarz and Hölder inequalities imply

$$
V_{2}(t, x) \leqslant C\|h\|_{H}^{p} \mu\left(t-t_{n}\right)^{p / 2}\left(1+\sup _{(t, x) \in[0, T] \times \mathbb{R}^{2}} \mathrm{E}\left(|X(t, x)|^{p}\right)\right) \leqslant C 2^{-n(\beta+1) p / 2} .
$$

Finally, Hölder's inequality implies, for $v(t)$ defined by (A.3):

$$
\begin{aligned}
V_{3}(t, x) & \leqslant C\left(\int_{t_{n}}^{t} \int_{\mathbb{R}^{2}} S(t-s, x-y) \mathrm{d} s \mathrm{~d} y\right)^{p}\left(1+\sup _{\substack{0 \leqslant t \leqslant T \\
x \in \mathbb{R}^{2}}} \mathrm{E}\left(|X(t, x)|^{p}\right)\right) \\
& \leqslant C v\left(t-t_{n}\right)^{p} \leqslant C 2^{-2 n p},
\end{aligned}
$$

which completes the proof of (2.17). Finally, (2.18) is a straightforward consequence of (A.15) and (2.17).

Consider the Picard iteration scheme associated with (2.1):

$$
\begin{aligned}
X_{n}^{0}(t, x)= & X^{0}(t, x), \\
X_{n}^{k+1}(t, x)= & X^{0}(t, x)+\int_{0}^{t} \int_{\mathbb{R}^{2}} S(t-s, x-y) A\left(X_{n}^{k}(s, y)\right) F(\mathrm{~d} s, \mathrm{~d} y) \\
& +\left\langle S(t-\cdot, x-*) B\left(X_{n}^{k}(\cdot, *)\right), \omega^{n}\right\rangle_{H}+\left\langle S(t-\cdot, x-*) D\left(X_{n}^{k}(\cdot, *)\right), h\right\rangle_{H} \\
& +\int_{0}^{t} \int_{\mathbb{R}^{2}} S(t-s, x-y) b\left(X_{n}^{k}(s, y)\right) \mathrm{d} s \mathrm{~d} y, \quad k \geqslant 0 .
\end{aligned}
$$

For any $0 \leqslant r \leqslant t \leqslant T$ and every integer $k \geqslant 0$, set 


$$
\begin{aligned}
X_{n}^{0}(t, r ; x)= & X^{0}(t, x), \\
X_{n}^{k+1}(t, r ; x)= & X^{0}(t, x)+\int_{0}^{r} \int_{\mathbb{R}^{2}} S(t-s, x-y) A\left(X_{n}^{k}(s, y)\right) F(\mathrm{~d} s, \mathrm{~d} y) \\
& +\left\langle S(t-\cdot, x-*) B\left(X_{n}^{k}(\cdot, *)\right) 1_{[0, r]}(\cdot), \omega^{n}\right\rangle_{H} \\
& +\left\langle S(t-\cdot, x-*) D\left(X_{n}^{k}(\cdot, *)\right) 1_{[0, r]}(\cdot), h\right\rangle_{H} \\
& +\int_{0}^{r} \int_{\mathbb{R}^{2}} S(t-s, x-y) b\left(X_{n}^{k}(s, y)\right) \mathrm{d} s \mathrm{~d} y, \\
\bar{X}_{n}^{k+1}(t, x)= & X_{n}^{k+1}\left(t, t_{n} ; x\right) .
\end{aligned}
$$

Notice that $X_{n}^{k}(t, t ; x)=X_{n}^{k}(t, x)$.

Lemma 2.3. Assume (C1), (C2) and (C3'). Then, for every $p \in[1, \infty), t \in[0, T], k \geqslant 1$, $n \geqslant 1$,

$$
\begin{aligned}
& \sup _{(s, y) \in[0, t] \times \mathbb{R}^{2}} \mathrm{E}\left(\left|X_{n}^{k}(s, y)-\bar{X}_{n}^{k}(s, y)\right|^{p} 1_{A_{n}(s)}\right) \leqslant C n^{p} 2^{-n(1+\beta) p / 2} \\
& \times\left[1+\sup _{(s, y) \in[0, t] \times \mathbb{R}^{2}} \mathrm{E}\left(\left|X_{n}^{k-1}(s, y)\right|^{p} 1_{A_{n}(s)}\right]\right.
\end{aligned}
$$

and

$$
\begin{aligned}
& \sup _{(s, y) \in[0, t] \times \mathbb{R}^{2}} \mathrm{E}\left(\left|X_{n}(s, y)-X_{n}^{-}(s, y)\right|^{p} 1_{A_{n}(s)}\right) \leqslant C n^{p} 2^{-n(1+\beta) p / 2} \\
& \quad \times\left[1+\sup _{(s, y) \in[0, t] \times \mathbb{R}^{2}} \mathrm{E}\left(\left|X_{n}(s, y)\right|^{p} 1_{A_{n}(s)}\right)\right] .
\end{aligned}
$$

Proof. Consider the decomposition

$$
\mathrm{E}\left(\left|X_{n}^{k}(t, x)-\bar{X}_{n}^{k}(t, x)\right|^{p} 1_{A_{n}(t)}\right) \leqslant C \sum_{i=1}^{4} T_{n}^{k, i}(t, x),
$$

with

$$
\begin{aligned}
& T_{n}^{k, 1}(t, x)=\mathrm{E}\left(\left|\int_{t_{n}}^{t} \int_{\mathbb{R}^{2}} S(t-s, x-y) A\left(X_{n}^{k-1}(s, y)\right) F(\mathrm{~d} s, \mathrm{~d} y)\right|^{p} 1_{A_{n}(t)}\right), \\
& T_{n}^{k, 2}(t, x)=\mathrm{E}\left(\left|\left\langle S(t-\cdot, x-*) B\left(X_{n}^{k-1}(\cdot, *)\right) 1_{\left(t_{n}, t\right]}(\cdot), \omega^{n}\right\rangle_{H}\right|^{p} 1_{A_{n}(t)}\right), \\
& T_{n}^{k, 3}(t, x)=\mathrm{E}\left(\left|\left\langle S(t-\cdot, x-*) D\left(X_{n}^{k-1}(\cdot, *)\right) 1_{\left(t_{n}, t\right]}(\cdot), h\right\rangle_{H}\right|^{p} 1_{A_{n}(t)}\right), \\
& T_{n}^{k, 4}(t, x)=\mathrm{E}\left(\left|\int_{t_{n}}^{t} \int_{\mathbb{R}^{2}} S(t-s, x-y) b\left(X_{n}^{k-1}(s, y)\right) \mathrm{d} s \mathrm{~d} y\right|^{p} 1_{A_{n}(t)}\right) .
\end{aligned}
$$


The local property of stochastic integrals, the inclusion $A_{n}(s) \supset A_{n}(t)$ for $s \leqslant t$, Burkholder's and Hölder's inequalities and (A.11) yield

$$
\begin{aligned}
T_{n}^{k, 1}(t, x) & \leqslant C \mu\left(t-t_{n}\right)^{p / 2}\left[1+\sup _{(s, y) \in[0, t] \times \mathbb{R}^{2}} \mathrm{E}\left(\left|X_{n}^{k-1}(s, y)\right|^{p} 1_{A_{n}(s)}\right)\right] \\
& \leqslant C 2^{-n(\beta+1) p / 2}\left[1+\sup _{(s, y) \in[0, t] \times \mathbb{R}^{2}} \mathrm{E}\left(\left|X_{n}^{k-1}(s, y)\right|^{p} 1_{A_{n}(s)}\right)\right] .
\end{aligned}
$$

The Cauchy-Schwarz and Hölder inequalities, along with (2.13) and (A.11), imply

$$
\begin{aligned}
T_{n}^{k, 2}(t, x) & \leqslant \mathrm{E}\left[\left\|\omega^{n} 1_{\left[t_{n}, t\right]} 1_{A_{n}(t)}\right\|_{H}^{p}\left\|S(t-\cdot, x-*) B\left(X_{n}^{k-1}(\cdot, *)\right) 1_{\left[t_{n}, t\right]}(\cdot) 1_{A_{n}(t)}\right\|_{H}^{p}\right] \\
& \leqslant C n^{p} 2^{-n(1+\beta) p / 2}\left[1+\sup _{(s, y) \in[0, t] \times \mathbb{R}^{2}} \mathrm{E}\left(\left|X_{n}^{k-1}(s, y)\right|^{p} 1_{A_{n}(s)}\right)\right]
\end{aligned}
$$

Similarly, using (A.3) for the last inequality, we have

$$
\begin{aligned}
& T_{n}^{k, 3}(t, x) \leqslant C\|h\|_{H}^{p} 2^{-n(1+\beta) p / 2}\left[1+\sup _{(s, y) \in[0, t] \times \mathbb{R}^{2}} \mathrm{E}\left(\left|X_{n}^{k-1}(s, y)\right|^{p} 1_{A_{n}(s)}\right)\right], \\
& T_{n}^{k, 4}(t, x) \leqslant C 2^{-2 n p}\left[1+\sup _{(s, y) \in[0, t] \times \mathbb{R}^{2}} \mathrm{E}\left(\left|X_{n}^{k-1}(s, y)\right|^{p} 1_{A_{n}(s)}\right)\right] .
\end{aligned}
$$

Thus (2.24)-(2.28) conclude the proof of (2.22).

Similar computations can be carried out to prove upper estimates of the $p$ moments of the increments $\left|X_{n}^{k+1}(s, x)-X_{n}^{k}(s, x)\right| 1_{A_{n}(s)}$ and $\left|\bar{X}_{n}^{k+1}(s, x)-\bar{X}_{n}^{k}(s, x)\right| 1_{A_{n}(s)}$; they yield

$$
\lim _{k \rightarrow \infty} \sup _{(s, x) \in[0, t] \times \mathbb{R}^{2}} \mathrm{E}\left[\left(\left|X_{n}^{k}(s, x)-X_{n}(s, x)\right|^{p}+\left|\bar{X}_{n}^{k}(s, x)-X_{n}^{-}(s, x)\right|^{p}\right) 1_{A_{n}(s)}\right]=0 .
$$

Therefore, (2.22) and (2.29) yield (2.23).

We now prove the convergence of $X_{n}^{-}(s, y)$ to $X_{n}(s, y)$.

Lemma 2.4. Assume (C1), (C2) and $\left(\mathrm{C}^{\prime}\right)$. Then, for any $p \in[1,+\infty)$,

$$
\sup _{n \geqslant 1} \sup _{(t, x) \in[0, T] \times \mathbb{R}^{2}} \mathrm{E}\left[1_{A_{n}(t)}\left(\left|X_{n}(t, x)\right|^{p}+\left|X_{n}^{-}(t, x)\right|^{p}\right)\right]<\infty
$$

and

$$
\sup _{(t, x) \in[0, T] \times \mathbb{R}^{2}}\left\|\left(X_{n}(t, x)-X_{n}^{-}(t, x)\right) 1_{A_{n}(t)}\right\|_{p} \leqslant C n 2^{-n(1+\beta) / 2} .
$$

Proof. We want to show that, for any $p \in[1, \infty)$,

$$
\sup _{n \geqslant 1} \sup _{k \geqslant 0} \sup _{(t, x) \in[0, T] \times \mathbb{R}^{2}} \mathrm{E}\left[1_{A_{n}(t)}\left(\left|X_{n}^{k}(t, x)\right|^{p}+\left|\bar{X}_{n}^{k}(t, x)\right|^{p}\right)\right]<+\infty .
$$

Indeed, (2.30) is a consequence of (2.32) and (2.29). For $r \leqslant t$, consider the decomposition 


$$
\mathrm{E}\left(\left|X_{n}^{k+1}(t, r ; x)\right|^{p} 1_{A_{n}(t)}\right) \leqslant C \sum_{i=1}^{6} T_{n}^{k+1, i}(t, r ; x)
$$

where

$$
\begin{aligned}
& T_{n}^{k+1,1}(t, r ; x)=\left|X^{0}(t, x)\right|^{p}, \\
& T_{n}^{k+1,2}(t, r ; x)=\mathrm{E}\left(\left|\int_{0}^{r} \int_{\mathbb{R}^{2}} S(t-s, x-y) A\left(X_{n}^{k}(s, y)\right) F(\mathrm{~d} s, \mathrm{~d} y)\right|^{p} 1_{A_{n}(t)}\right), \\
& T_{n}^{k+1,3}(t, r ; x)=\mathrm{E}\left(\left|\left\langle S(t-\cdot, x-*) B\left(\bar{X}_{n}^{k}(\cdot, *)\right) 1_{[0, r]}(\cdot), \omega^{n}\right\rangle_{H}\right|^{p} 1_{A_{n}(t)}\right), \\
& T_{n}^{k+1,4}(t, r ; x)=\mathrm{E}\left(\left|\left\langle S(t-\cdot, x-*)\left[B\left(X_{n}^{k}\right)-B\left(\bar{X}_{n}^{k}\right)\right](\cdot, *) 1_{[0, r]}(\cdot), \omega^{n}\right\rangle_{H}\right|^{p} 1_{A_{n}(t)}\right), \\
& T_{n}^{k+1,5}(t, r ; x)=\mathrm{E}\left(\left|\left\langle S(t-\cdot, x-*) D\left(X_{n}^{k}\right)(\cdot, *) 1_{[0, r]}(\cdot), h\right\rangle_{H}\right|^{p} 1_{A_{n}(t)}\right), \\
& T_{n}^{k+1,6}(t, r ; x)=\mathrm{E}\left(\left|\int_{0}^{r} \int_{\mathbb{R}^{2}} S(t-s, x-y) b\left(X_{n}^{k}(s, y)\right) \mathrm{d} s \mathrm{~d} y\right|^{p} 1_{A_{n}(t)}\right) .
\end{aligned}
$$

Under hypotheses weaker than (C2), we proved in Millet and Sanz-Solé (1999) that

$$
\left|X^{0}(t, x)\right| \leqslant C\left(\left\|v_{0}\right\|_{q_{0}}+\left\|\nabla u_{0}\right\|_{q_{0}}\right)
$$

Burkholder's and Hölder's inequalities yield

$$
T_{n}^{k+1,2}(t, r ; x) \leqslant C \int_{0}^{r} J(t-s)\left[1+\sup _{(u, y) \in[0, s] \times \mathbb{R}^{2}} \mathrm{E}\left(\left|X_{n}^{k}(u, y)\right|^{p} 1_{A_{n}(u)}\right)\right] \mathrm{d} s .
$$

Let $\tau_{n}$ be the operator defined on real-valued functions by

$$
\tau_{n}(\rho)(s, x)=\rho\left(\left(s+2^{-n}\right) \wedge T, x\right) .
$$

Let $\mathscr{E}_{n}$ be the closed subspace of $H$ generated by the orthonormal system

$$
2^{n} T^{-1} 1_{\Delta_{i}}(\cdot) \otimes e_{j}(*), \quad i=0, \ldots, 2^{n}-1, j=1, \ldots, n,
$$

and denote by $\pi_{n}$ the orthogonal projection operator on $\mathscr{E}_{n}$. Then since $\bar{X}_{n}^{k}(s, y)$ is $\mathscr{F}_{s_{n}}$ measurable, the definition of $\omega_{n}$ in (1.11) implies

$T_{n}^{k+1,3}(t, r ; x)=\mathrm{E}\left(\left|\int_{0}^{1} \int_{\mathbb{R}^{2}}\left(\pi_{n} \circ \tau_{n}\right)\left[S(t-\cdot, x-*) B\left(\bar{X}_{n}^{k}(\cdot, *)\right) 1_{[0, r]}(\cdot) 1_{A_{n}(\cdot)}\right](s, y) F(\mathrm{~d} s, \mathrm{~d} y)\right|^{p}\right)$

and, by Burkholder's and Hölder's inequalities, if $J$ is defined by (A.1),

$$
\begin{aligned}
T_{n}^{k+1,3}(t, r ; x) & \leqslant C \mathrm{E} \|\left(\pi_{n} \circ \tau_{n}\right)\left(S(t-\cdot, x-*) B\left(\bar{X}_{n}^{k}(\cdot, *) 1_{[0, r]}(\cdot) 1_{A_{n}(\cdot)}\right) \|_{H}^{p}\right. \\
& \leqslant C \int_{0}^{r} J(t-s)\left[1+\sup _{(u, y) \in[0, s] \times \mathbb{R}^{2}} \mathrm{E}\left(\left|\bar{X}_{n}^{k}(u, y)\right|^{p} 1_{A_{n}(u)}\right)\right] \mathrm{d} s .
\end{aligned}
$$


The Cauchy-Schwarz and Hölder inequalities, along with (2.11) and (2.22), imply

$$
\begin{aligned}
T_{n}^{k+1,4}(t, r ; x) & \leqslant \mathrm{E}\left(\left\|\omega^{n} 1_{A_{n}(t)}\right\|_{H}^{p}\left\|S(t-\cdot, x-*)\left[B\left(X_{n}^{k}\right)-B\left(\bar{X}_{n}^{k}\right)\right](\cdot, *) 1_{[0, r]}(\cdot) 1_{A_{n}(t)}\right\|_{H}^{p}\right) \\
& \leqslant C n^{p} 2^{n p / 2} \int_{0}^{r} J(t-s) \sup _{y \in \mathbb{R}^{2}} \mathrm{E}\left(\left|X_{n}^{k}(s, y)-\bar{X}_{n}^{k}(s, y)\right|^{p} 1_{A_{n}(t)}\right) \mathrm{d} s \\
& \leqslant C n^{2 p} 2^{-n \beta n p / 2} \int_{0}^{r} J(t-s)\left[1+\sup _{(u, y) \in[0, s] \times \mathbb{R}^{2}} \mathrm{E}\left(\left|X_{n}^{k-1}(u, y)\right|^{p} 1_{A_{n}(u)}\right)\right] \mathrm{d} s .
\end{aligned}
$$

Analogously,

$$
\begin{aligned}
& T_{n}^{k+1,5}(t, r ; x) \leqslant C\|h\|_{H}^{p} \int_{0}^{r} J(t-s)\left[1+\sup _{(u, y) \in[0, s] \times \mathbb{R}^{2}} \mathrm{E}\left(\left|X_{n}^{k}(u, y)\right|^{p} 1_{A_{n}(u)}\right)\right] \mathrm{d} s, \\
& T_{n}^{k+1,6}(t, r ; x) \leqslant C \int_{0}^{r}(t-s)\left[1+\sup _{(u, y) \in[0, s] \times \mathbb{R}^{2}} \mathrm{E}\left(\left|X_{n}^{k}(u, y)\right|^{p} 1_{A_{n}(u)}\right)\right] \mathrm{d} s .
\end{aligned}
$$

Therefore, (2.33)-(2.39) yield

$$
\begin{aligned}
\mathrm{E}\left(\left|X_{n}^{k+1}(t, r ; x)\right|^{p} 1_{A_{n}(t)}\right) \leqslant \int_{0}^{r} & {\left[1+\sup _{(u, y) \in[0, s] \times \mathbb{R}^{2}}\left\{\mathrm { E } \left(\left[\left|X_{n}^{k}(u, y)\right|^{p}\right.\right.\right.\right.} \\
& \left.\left.\left.\left.+\left|X_{n}^{k-1}(u, y)\right|^{p}+\left|\bar{X}_{n}^{k}(u, y)\right|^{p}\right] 1_{A_{n}(u)}\right)\right\}\right] \mathrm{d} s .
\end{aligned}
$$

Set, for any $k \geqslant 0, t \in[0, T]$,

$$
\varphi_{n}^{k}(t)=\sup _{(s, y) \in[0, t] \times \mathbb{R}^{2}} \mathrm{E}\left(\left(\left|X_{n}^{k}(s, y)\right|^{p}+\left|\bar{X}_{n}^{k}(s, y)\right|^{p}\right) 1_{A_{n}(s)}\right) .
$$

Then, using (2.40) with $r=t$ and $r=t_{n}$ and adding term by term the corresponding inequalities, we obtain

$$
\varphi_{n}^{k+1}(t) \leqslant C \int_{0}^{t}\left[1+\varphi_{n}^{k}(s)+\varphi_{n}^{k-1}(s)\right] \mathrm{d} s,
$$

with the convention $\varphi_{n}^{-1}(\cdot) \equiv 0$. Since, by $(2.34)$,

$$
\varphi_{n}^{0}(t) \leqslant 2 \sup _{x \in \mathbb{R}^{2}}\left|X^{0}(t, x)\right|^{p} \leqslant C,
$$

(2.41) yields

$$
\sup _{n \geqslant 1} \sup _{k \geqslant 0} \sup _{t \in[0, T]} \varphi_{n}^{k}(t) \leqslant C,
$$


which establishes (2.32). Finally, inequalities (2.23) and (2.30) inply (2.31), which completes the proof of the lemma.

In what follows, $K$ denotes an arbitrary compact subset of $\mathbb{R}^{2}$. For any $s, t, \bar{t} \in[0, T]$, $x, \bar{x} \in K, y \in \mathbb{R}^{2}$, set

$$
\gamma(t, \bar{t}, x, \bar{x} ; s, y)=S(t-s, x-y)-S(\bar{t}-s, \bar{x}-y)
$$

and

$$
\Gamma(t, \bar{t}, x, \bar{x} ; s, y)=|\gamma(t, \bar{t}, x, \bar{x} ; s, y)| .
$$

Lemma 2.5. Assume that the function $f$ satisfies condition (C1). For any $0<\gamma<$ $\beta /(2(1+\beta)), t, \bar{t} \in[0, T], x, \bar{x} \in K$,

$$
\begin{gathered}
\|\Gamma(t, \bar{t}, x, \bar{x} ; \cdot, *)\|_{H} \leqslant C\left(|t-\bar{t}|^{\gamma}+|x-\bar{x}|^{\gamma}\right), \\
\int_{0}^{T} \int_{\mathbb{R}^{2}} \Gamma(t, \bar{t}, x, \bar{x} ; s, y) \mathrm{d} s \mathrm{~d} y \leqslant C\left(|t-\bar{t}|^{1 / 2}+|x-\bar{x}|^{1 / 2}\right) .
\end{gathered}
$$

Proof. Assume $0 \leqslant t \leqslant \bar{t} \leqslant T$ and set

$$
\begin{aligned}
& \Gamma_{1}(t, \bar{t}, x, \bar{x} ; s, y)=(S(t-s, x-y)-S(\bar{t}-s, x-y)) 1_{[0, t]}(s), \\
& \Gamma_{2}(t, \bar{t}, x, \bar{x} ; s, y)=\left|(S(\bar{t}-s, x-y)-S(\bar{t}-s, \bar{x}-y)) 1_{[0, t]}(s)\right|, \\
& \Gamma_{3}(t, \bar{t}, x, \bar{x} ; s, y)=S(\bar{t}-s, \bar{x}-y) 1_{[t, \bar{t}]}(s)
\end{aligned}
$$

then

$$
\|\Gamma(t, \bar{t}, x, \bar{x} ; \cdot, *)\|_{H}^{2} \leqslant C \sum_{i=1}^{3}\left\|\Gamma_{i}(t, \bar{t}, x, \bar{x} ; \cdot, *)\right\|_{H}^{2} .
$$

For $i=1,2$, it is easy to check that

$$
\begin{gathered}
\left\|\Gamma_{1}(t, \bar{t}, x, \bar{x} ; \cdot, *)\right\|_{H}^{2} \leqslant \mu_{t, \bar{t}-t}+\tilde{\mu}_{t, \bar{t}-t}+2\left(\mu_{t, \bar{t}-t} \tilde{\mu}_{t, \bar{t}-t}\right)^{1 / 2}, \\
\left\|\Gamma_{2}(t, \bar{t}, x, \bar{x} ; \cdot \cdot *)\right\|_{H}^{2} \leqslant M_{t, \bar{x}-x}+N_{t, \bar{x}-x}+2\left(M_{t, \bar{x}-x} N_{t, \bar{x}-x}\right)^{1 / 2},
\end{gathered}
$$

where $\mu_{t, \bar{t}-t}, \tilde{\mu}_{t, \bar{t}-t}, M_{t, \bar{x}-x}, N_{t, \bar{x}-x}$ are defined in (A.6)-(A.9), respectively. Finally, for $\mu(t)$ defined by (A.2),

$$
\left\|\Gamma_{3}(t, \bar{t}, x, \bar{x} ; \cdot, *)\right\|_{H}^{2}=\mu(\bar{t}-t) .
$$

Thus the estimates (A.11)-(A.13) show (2.42).

In order to prove $(2.43)$, set $\Gamma(t, \bar{t}, x, \bar{x} ; s, y) \leqslant \sum_{i=1}^{2} \bar{\Gamma}_{i}(t, \bar{t}, x, \bar{x} ; s, y)$, with 


$$
\begin{aligned}
& \bar{\Gamma}_{1}(t, \bar{t}, x, \bar{x} ; s, y)=|S(t-s, x-y)-S(\bar{t}-s, x-y)|, \\
& \bar{\Gamma}_{2}(t, \bar{t}, x, \bar{x} ; s, y)=|S(\bar{t}-s, x-y)-S(\bar{t}-s, \bar{x}-y)| .
\end{aligned}
$$

Assume $0 \leqslant t \leqslant \bar{t} \leqslant T$; then

$$
\int_{0}^{T} \mathrm{~d} s \int_{\mathbb{R}^{2}} \mathrm{~d} y \bar{\Gamma}_{1}(t, \bar{t}, x, \bar{x} ; s, y) \leqslant C\left(v_{t, \bar{t}-t}+\tilde{v}_{t, \bar{t}-t}+v(\bar{t}-t)\right)
$$

with $v(\bar{t}-t), v_{t, \bar{t}-t}, \tilde{v}_{t, \bar{t}-t}$, defined in (A.3)-(A.5). Hence, inequalities (A.3) and (A.10) imply

$$
\int_{0}^{T} \mathrm{~d} s \int_{\mathbb{R}^{2}} \mathrm{~d} y \bar{\Gamma}_{1}(t, \bar{t}, x, \bar{x} ; s, y) \leqslant C(\bar{t}-t)^{1 / 2}
$$

Moreover,

$$
\int_{0}^{T} \mathrm{~d} s \int_{\mathbb{R}^{2}} \mathrm{~d} y \bar{\Gamma}_{2}(t, \bar{t}, x, \bar{x} ; s, y) \leqslant \bar{\Gamma}_{2,1}(t, \bar{t}, x, \bar{x})+2 \bar{\Gamma}_{2,2}(t, \bar{t}, x, \bar{x}),
$$

with

$$
\begin{aligned}
& \bar{\Gamma}_{2,1}(t, \bar{t}, x, \bar{x})=\int_{0}^{\bar{t}-\frac{|x-\bar{x}|}{2}} \mathrm{~d} s \int_{\substack{|x-y| \bar{t}-s \\
|\bar{x}-y| \bar{t}-s}} \mathrm{~d} y|S(\bar{t}-s, x-y)-S(\bar{t}-s, \bar{x}-y)|, \\
& \bar{\Gamma}_{2,2}(t, \bar{t}, x, \bar{x})=\int_{0}^{\bar{t}} \mathrm{~d} s \int_{\substack{|\overline{\mid}-y|<\overline{-}-s \\
|x-y| \geq \bar{t}-s}} \mathrm{~d} y S(\bar{t}-s, \bar{x}-y) .
\end{aligned}
$$

Using (A.14), we obtain $\bar{\Gamma}_{2,1}(t, \bar{t}, x, \bar{x}) \leqslant C|x-\bar{x}|^{1 / 2}$. Finally, an explicit computation implies $\bar{\Gamma}_{2,2}(t, \bar{t}, x, \bar{x}) \leqslant C|x-\bar{x}|^{1 / 2}$. Thus

$$
\int_{0}^{T} \mathrm{~d} s \int_{\mathbb{R}^{2}} \mathrm{~d} y \bar{\Gamma}_{2}(t, \bar{t}, x, \bar{x} ; s, y) \leqslant C|x-\bar{x}|^{1 / 2} .
$$

Inequalities (2.44) and (2.45) show (2.43) and conclude the proof of the lemma.

In the next proposition, we show that the sequence of processes $\left\{X_{n}(t, x), n \geqslant 1\right\}$ satisfies assumption (P1) of Lemma A.2. It proves estimates similar to those in Millet and Sanz-Solé (1999, Proposition 1.4) which are uniform in $n$.

Proposition 2.2. Assume (C1), (C2), (C3'). For any $p \in[1, \infty), 0 \leqslant t \leqslant \bar{t} \leqslant T, x, \bar{x} \in K$, $\gamma \in] 0, \beta /(2(1+\beta))[$,

$$
\sup _{n}\left\|\left(X_{n}(t, x)-X_{n}(\bar{t}, \bar{x})\right) 1_{A_{n}(\bar{t})}\right\|_{p} \leqslant C\left(|t-\bar{r}|^{\gamma}+|x-\bar{x}|^{\gamma}\right) .
$$


Proof. Consider the decomposition

$$
\mathrm{E}\left(\left|X_{n}(t, x)-X_{n}(\bar{t}, \bar{x})\right|^{p} 1_{A_{n}(\bar{t})}\right) \leqslant C \sum_{i=1}^{6} R_{n}^{i}(t, \bar{t} ; x, \bar{x}),
$$

where

$$
\begin{aligned}
& R_{n}^{1}(t, \bar{t} ; x, \bar{x})=\left|X_{0}(t, x)-X_{0}(\bar{t}, \bar{x})\right|^{p} \\
& R_{n}^{2}(t, \bar{t} ; x, \bar{x})=\mathrm{E}\left(\left|\int_{0}^{T} \int_{\mathbb{R}^{2}} \gamma(t, \bar{t}, x, \bar{x} ; s, y) A\left(X_{n}(s, y)\right) F(\mathrm{~d} s, \mathrm{~d} y)\right|^{p} 1_{A_{n}(\bar{t})}\right), \\
& R_{n}^{3}(t, \bar{t} ; x, \bar{x})=\mathrm{E}\left(\left|\left\langle\gamma(t, \bar{t}, x, \bar{x} ; \cdot, *) B\left(X_{n}^{-}(\cdot, *)\right), \omega^{n}\right\rangle\right|_{H}^{p} 1_{A_{n}(\bar{t})}\right), \\
& R_{n}^{4}(t, \bar{t} ; x, \bar{x})=\mathrm{E}\left(\left|\left\langle\gamma(t, \bar{t}, x, \bar{x} ; \cdot, *)\left(B\left(X_{n}\right)-B\left(X_{n}^{-}\right)\right)(\cdot, *), \omega^{n}\right\rangle\right|_{H}^{p} 1_{A_{n}(\bar{t})}\right), \\
& R_{n}^{5}(t, \bar{t} ; x, \bar{x})=\mathrm{E}\left(\left|\left\langle\gamma(t, \bar{t}, x, \bar{x} ; \cdot, *) D\left(X_{n}(\cdot, *)\right), h\right\rangle\right|_{H}^{p} 1_{A_{n}(\bar{t})}\right), \\
& R_{n}^{6}(t, \bar{t} ; x, \bar{x})=\mathrm{E}\left(\left|\int_{0}^{T} \int_{\mathbb{R}^{2}} \gamma(t, \bar{t}, x, \bar{x} ; s, y) b\left(X_{n}(s, y)\right) \mathrm{d} s \mathrm{~d} y\right|^{p} 1_{A_{n}(\bar{t})}\right) .
\end{aligned}
$$

In the proof of Proposition 1.4 in Millet and Sanz-Solé (1999) we checked that (C2) implies that

$$
R_{n}^{1}(t, \bar{t} ; x, \bar{x}) \leqslant C\left(|t-\bar{t}|^{\delta}+|x-\bar{x}|^{\delta}\right)
$$

for $\delta=\beta /(2(1+\beta))$. Burkholder's and Hölder's inequalities yield

$$
R_{n}^{2}(t, \bar{t} ; x, \bar{x}) \leqslant C\|\Gamma(t, \bar{t}, x, \bar{x} ; \cdot, *)\|_{H}^{p}\left[1+\sup _{(s, y) \in[0, T] \times \mathbb{R}^{2}} \mathrm{E}\left(\left|X_{n}(s, y)\right|^{p} 1_{A_{n}(t)}\right)\right] .
$$

Using the operators $\tau_{n}$ and $\pi_{n}$ introduced in the proof of Lemma 2.4, we may rewrite the scalar product appearing in $R_{n}^{3}$ as a stochastic integral; thus Burkholder's inequality implies

$$
\begin{aligned}
R_{n}^{3}(t, \bar{t} ; x, \bar{x}) & =\mathrm{E}\left(\left|\int_{0}^{T} \int_{\mathbb{R}^{2}}\left(\pi_{n} \circ \tau_{n}\right)\left(\Gamma(t, \bar{t}, x, \bar{x} ; \cdot, *) B\left(X_{n}^{-}(\cdot, *)\right) 1_{A_{n}(\bar{t})}\right)(s, y) F(\mathrm{~d} s, \mathrm{~d} y)\right|^{p}\right) \\
& \leqslant C\|\Gamma(t, \bar{t}, x, \bar{x} ; \cdot, *)\|_{H}^{p}\left[1+\sup _{(s, y) \in[0, T] \times \mathbb{R}^{2}} \mathrm{E}\left(\left|X_{n}^{-}(s, y)\right|^{p} 1_{A_{n}(s)}\right)\right] .
\end{aligned}
$$

The Cauchy-Schwarz and Hölder inequalities, along with (2.11) and (2.31), imply 


$$
\begin{aligned}
R_{n}^{4}(t, \bar{t} ; x, \bar{x}) & \leqslant\left\{\mathrm{E}\left(\left\|\omega_{n}\right\|_{H}^{2 p} 1_{A_{n}(T)}\right) \mathrm{E}\left(\left\|\gamma(t, \bar{t}, x, \bar{x} ; \cdot, *)\left(B\left(X_{n}\right)-B\left(X_{n}^{-}\right)\right)(\cdot, *)\right\|_{H}^{2 p} 1_{A_{n}(\bar{t})}\right)\right\}^{1 / 2} \\
& \leqslant C n^{p} 2^{n p / 2}\|\Gamma(t, \bar{t}, x, \bar{x} ; \cdot, *)\|_{H}^{p}\left\{\sup _{(s, y) \in[0, T] \times \mathbb{R}^{2}} \mathrm{E}\left(\left|X_{n}(s, y)-X_{n}^{-}(s, y)\right|^{2 p} 1_{A_{n}(s)}\right)\right\}^{1 / 2} \\
& \leqslant C n^{2 p} 2^{-n \beta p / 2}\|\Gamma(t, \bar{t}, x, \bar{x} ; \cdot, *)\|_{H}^{p} .
\end{aligned}
$$

Finally

$$
\begin{aligned}
& R_{n}^{5}(t, \bar{t} ; x, \bar{x}) \leqslant C\|h\|_{H}^{p}\|\Gamma(t, \bar{t}, x, \bar{x} ; \cdot, *)\|_{H}^{p}\left[1+\sup _{(s, y) \in[0, T] \times \mathbb{R}^{2}} \mathrm{E}\left(\left|X_{n}(s, y)\right|^{p} 1_{A_{n}(s)}\right)\right], \\
& R_{n}^{6}(t, \bar{t} ; x, \bar{x}) \leqslant C\left(\int_{0}^{T} \int_{\mathbb{R}^{2}} \Gamma(t, \bar{t}, x, \bar{x} ; s, y) \mathrm{d} s \mathrm{~d} y\right) p\left[1+\sup _{(s, y) \in[0, T] \times \mathbb{R}^{2}} \mathrm{E}\left(\left|X_{n}(s, y)\right|^{p} 1_{A_{n}(s)}\right)\right] .
\end{aligned}
$$

Hence, (2.46)-(2.51), (2.30) and Lemma 2.5 yield the proposition.

Remark 2.2. Proposition 2.2 establishes the $\gamma$-Hölder continuity for the trajectories of $X_{n}$ on $A_{n}(T)$, because the sets $A_{n}(t), t \in[0, T]$, are decreasing. In Lemma 2.1 we have shown $\lim _{\alpha \rightarrow \infty} P\left(A_{n}(T)\right)=1$, so that the trajectories of $X_{n}$ are almost surely $\gamma$-Hölder continuous on $[0, T] \times K$ for any $\gamma<\beta / 2(1+\beta)$ and any compact subset $K$ of $\mathbb{R}^{2}$.

We now prove that the processes $\left\{X_{n}(t, x), n \geqslant 1\right\}$ satisfy condition (P2) of Lemma A2.

Proposition 2.3. Suppose that conditions (C1), (C2) and (C3') are satisfied. Then, for any $p \in[1, \infty),(t, x) \in[0, T] \times K$,

$$
\lim _{n \rightarrow \infty}\left\|\left(X_{n}(t, x)-X(t, x)\right) 1_{A_{n}(t)}\right\|_{p}=0 .
$$

Proof. Set

$$
X_{n}(t, x)-X(t, x)=\sum_{i=1}^{8} U_{n}^{i}(t, x),
$$

where 


$$
\begin{aligned}
& U_{n}^{1}(t, x)=\int_{0}^{t} \int_{\mathbb{R}^{2}} S(t-s, x-y)\left[(A+B)\left(X_{n}(s, y)\right)-(A+B)(X(s, y))\right] F(\mathrm{~d} s, \mathrm{~d} y), \\
& U_{n}^{2}(t, x)=\left\langle S(t-\cdot, x-*)\left[D\left(X_{n}(\cdot, *)\right)-D(\mathrm{X}(\cdot, *))\right], h\right\rangle_{H}, \\
& U_{n}^{3}(t, x)=\int_{0}^{t} \int_{\mathbb{R}^{2}} S(t-s, x-y)\left[b\left(X_{n}(s, y)\right)-b(X(s, y))\right] \mathrm{d} s \mathrm{~d} y, \\
& U_{n}^{4}(t, x)=\left\langle S(t-\cdot, x-*)\left[B\left(X_{n}(\cdot, *)\right)-B\left(X_{n}^{-}(\cdot, *)\right)\right], \omega^{n}\right\rangle_{H}, \\
& U_{n}^{5}(t, x)=\left\langle S(t-\cdot, x-*)\left[B\left(X_{n}^{-}(\cdot, *)\right)-B\left(X^{-}(\cdot, *)\right)\right], \omega^{n}\right\rangle_{H}, \\
& U_{n}^{6}(t, x)=\left\langle S(t-\cdot, x-*) B\left(X^{-}(\cdot, *)\right), \omega^{n}\right\rangle_{H}-\int_{0}^{t} \int_{\mathbb{R}^{2}} S(t-s, x-y) B\left(X^{-}(s, y)\right) F(\mathrm{~d} s, \mathrm{~d} y), \\
& U_{n}^{7}(t, x)=\int_{0}^{t} \int_{\mathbb{R}^{2}} S(t-s, x-y)\left[B\left(X^{-}(s, y)\right)-B\left(X_{n}^{-}(s, y)\right)\right] F(\mathrm{~d} s \mathrm{~d} y), \\
& U_{n}^{8}(t, x)=\int_{0}^{t} \int_{\mathbb{R}^{2}} S(t-s, x-y)\left[B\left(X_{n}^{-}(s, y)\right)-B\left(X_{n}(s, y)\right)\right] F(\mathrm{~d} s \mathrm{~d} y),
\end{aligned}
$$

with $X_{n}^{-}$and $X^{-}$defined in (2.15) and (2.16), respectively.

For $t \in[0, T]$, let $K(t)=\left\{x \in \mathbb{R}^{2}: d(x, K) \leqslant T-t\right\}$; fix $t \in[0, T]$ and $x \in K(t)$. Burkholder's and Hölder's inequalities imply

$$
\left\|U_{n}^{1}(t, x) 1_{A_{n}(t)}\right\|_{p}^{p} \leqslant C \int_{0}^{t} J(t-s) \sup _{y \in K(s)}\left\|\left(X_{n}(s, y)-X(s, y)\right) 1_{A_{n}(s)}\right\|_{p}^{p} \mathrm{~d} s .
$$

The Cauchy-Schwarz and Hölder inequalities yield

$$
\left\|U_{n}^{2}(t, x) 1_{A_{n}(t)}\right\|_{p}^{p} \leqslant C\|h\|_{H}^{p} \int_{0}^{t} J(t-s) \sup _{y \in K(s)}\left\|\left(X_{n}(s, y)-X(s, y)\right) 1_{A_{n}(s)}\right\|_{p}^{p} \mathrm{~d} s .
$$

Analogously,

$$
\left\|U_{n}^{3}(t, x) 1_{A_{n}(t)}\right\|_{p}^{p} \leqslant C \int_{0}^{t}(t-s) \sup _{y \in K(s)}\left\|\left(X_{n}(s, y)-X(s, y)\right) 1_{A_{n}(s)}\right\|_{p}^{p} \mathrm{~d} s .
$$

Since

$$
U_{n}^{5}(t, x)=\int_{0}^{t} \int_{\mathbb{R}^{2}}\left(\pi_{n} \circ \tau_{n}\right)\left[S(t-\cdot, x-*)\left[B\left(X_{n}^{-}\right)-B\left(X^{-}\right)\right](\cdot, *) 1_{A_{n}(\cdot)}\right](s, y) F(\mathrm{~d} s, \mathrm{~d} y),
$$

Burkholder's and Hölder's inequalities easily yield

$$
\left\|\left(U_{n}^{5}(t, x)+U_{n}^{7}(t, x)\right) 1_{A_{n}(t)}\right\|_{p}^{p} \leqslant C \int_{0}^{t} J(t-s) \sup _{y \in K(s)}\left\|\left(X_{n}^{-}(s, y)-X^{-}(s, y)\right) 1_{A_{n}(s)}\right\|_{p}^{p} \mathrm{~d} s .
$$

Thus, (2.31) and (2.17) ensure that 


$$
\begin{aligned}
& \left\|\left(U_{n}^{5}(t, x)+U_{n}^{7}(t, x)\right) 1_{A_{n}(t)}\right\|_{p}^{p} \\
& \quad \leqslant C n^{p} 2^{-n(1+\beta) p / 2}+C \int_{0}^{t} J(t-s) \sup _{y \in K(s)}\left\|\left(X_{n}(s, y)-X(s, y)\right) 1_{A_{n}(s)}\right\|^{p} \mathrm{~d} s .
\end{aligned}
$$

Thus, by Gronwall's lemma, if suffices to check

$$
\sup _{0 \leqslant t \leqslant T} \sup _{x \in K(t)}\left\|U_{n}^{i}(t, x) 1_{A_{n}(t)}\right\|_{p} \underset{n \rightarrow \infty}{\rightarrow} 0, \quad i=4,6,8 .
$$

The Cauchy-Schwarz and Hölder inequalities, along with (2.11) and (2.31), imply that

$$
\begin{aligned}
& \left\|U_{n}^{4}(t, x) 1_{A_{n}(t)}\right\|_{p}^{p} \\
& \quad \leqslant C\left\{\mathrm{E}\left(\left\|\omega^{n} 1_{A_{n}(t)}\right\|_{H}^{2 p}\right)\right\}^{1 / 2} \sup _{(t, x) \in[0, T] \times \mathbb{R}^{2}}\left\{\mathrm{E}\left(\left|X_{n}(t, x)-X_{n}^{-}(t, x)\right|^{2 p} 1_{A_{n}(t)}\right)\right\}^{1 / 2} \\
& \quad \leqslant C n^{2 p} 2^{-n \beta p / 2} .
\end{aligned}
$$

Burkholder's and Hölder's inequalities and (2.31) imply

$$
\begin{aligned}
\left\|U_{n}^{8}(t, x) 1_{A_{n}(t)}\right\|_{p}^{p} & \leqslant C \int_{0}^{t} J(t-s) \sup _{x \in \mathbb{R}^{2}}\left(\left\|\left(X_{n}^{-}(s, x)-X_{n}(s, x)\right) 1_{A_{n}(s)}\right\|_{p}^{p}\right) \mathrm{d} s \\
& \leqslant C n^{p} 2^{-n(1+\beta) p / 2} ;
\end{aligned}
$$

thus (2.53) holds for $i=4,8$.

Set $\left\|U_{n}^{6}(t, x) 1_{A_{n}(t)}\right\|_{p}^{p} \leqslant C\left(U_{n}^{6,1}(t, x)+U_{n}^{6,2}(t, x)\right)$, with

$$
\begin{aligned}
& U_{n}^{6,1}(t, x)= \| \int_{0}^{t} \int_{\mathbb{R}^{2}}\left[\pi _ { n } \left(\left\{\tau_{n}\left(S(t-\cdot, x-*) B\left(X^{-}(\cdot, *)\right)\right)\right)\right.\right. \\
&\left.\left.\left.-S(t-\cdot, x-*) B\left(X^{-}(\cdot, *)\right)\right\} 1_{A_{n}(.)}\right)\right](s, y) F(\mathrm{~d} s, \mathrm{~d} y) \|_{p}^{p} \\
& U_{n}^{6,2}(t, x)=\| \int_{0}^{t} \int_{\mathbb{R}^{2}}\left[\pi_{n}\left(S(t-\cdot, x-*) B\left(X^{-}(\cdot, *)\right) 1_{A_{n}(\cdot)}\right)(s, y)\right. \\
&\left.-S(t-s, x-y) B\left(X^{-}(s, y)\right) 1_{A_{n}(s)}\right] F(\mathrm{~d} s, \mathrm{~d} y) \|_{p}^{p} .
\end{aligned}
$$

By Burkholder's inequality and the fact that $\pi_{n}$ is a contraction of $H$, we deduce that

$$
U_{n}^{6,1}(t, x) \leqslant C \mathrm{E}\left(\left\|\left[\tau_{n}\left(S(t-\cdot, x-*) B\left(X^{-}(\cdot, *)\right)\right)-S(t-\cdot, x-*) B\left(X^{-}(\cdot, *)\right)\right] 1_{A_{n}(\cdot)}\right\|_{H}^{p}\right) .
$$

Define 


$$
\begin{aligned}
& U_{n}^{6,1,1}(t, x)=\mathrm{E}\left(\left\|\left[\tau_{n}(S(t-\cdot, x-*))-S(t-\cdot, x-*)\right] \tau_{n}\left(B\left(X^{-}(\cdot, *)\right)\right) 1_{A_{n}(\cdot)}\right\|_{H}^{p}\right), \\
& U_{n}^{6,1,2}(t, x)=\mathrm{E}\left(\left\|S(t-\cdot, x-*) 1_{A_{n}(\cdot)}\left[\tau_{n}\left(B\left(X^{-}(\cdot, *)\right)\right)-B\left(X^{-}(\cdot, *)\right)\right]\right\|_{H}^{p}\right) .
\end{aligned}
$$

Expressions (2.18) and (A.12) together yield

$$
\sup _{(t, x) \in[0, T] \times \mathbb{R}^{2}} U_{n}^{6,1,1}(t, x) \leqslant C 2^{-n \gamma p}
$$

for any $\gamma<\beta / 2(1+\beta)$. Moreover, since $X$ is a particular case of $X_{n}$, by (2.17) and Proposition 2.2 we have

$$
\sup _{(s, x) \in[0, T] \times \mathbb{R}^{2}}\left\|\left(X^{-}\left(s+2^{-n}, x\right)-X^{-}(s, x)\right) 1_{A_{n}\left(s+2^{-n}\right)}\right\|_{p} \leqslant C 2^{-n \gamma}
$$

for $0<\gamma<\beta / 2(1+\beta)$. Furthermore, (2.18) yields that if $1 / p+1 / q=1$, then

$$
\sup _{(s, x) \in[0, T] \times \mathbb{R}^{2}}\left\|\left(\left|X^{-}\left(s+2^{-n}, x\right)\right|+\left|X^{-}(s, x)\right|\right) 1_{A_{n}(s) \backslash A_{n}\left(s+2^{-n}\right)}\right\| p \leqslant C\left[P\left(A_{n}(T)\right)^{c}\right]^{1 / q},
$$

so that, using Lemma 2.1 and (2.55), we conclude that

$$
\lim _{n} \sup _{(t, x) \in[0, T] \times \mathbb{R}^{2}} U_{n}^{6,1,2}(t, x)=0 .
$$

Inequalities (2.54) and (2.56) imply

$$
\lim _{n \rightarrow \infty} \sup _{(t, x) \in[0, T] \times \mathbb{R}^{2}} U_{n}^{6,1}(t, x)=0 .
$$

Let $I_{H}$ denote the identity operator on the Hilbert space $H$. Burkholder's inequality yields $U_{n}^{6,2}(t, x) \leqslant C Z_{n}(t, x)$, with, for $(t, x) \in[0, T] \times \mathbb{R}^{2}$,

$$
Z_{n}(t, x)=\mathrm{E}\left(\left\|\left(\pi_{n}-I_{H}\right)\left(S(t-\cdot, x-*) B\left(X^{-}(\cdot, *)\right) 1_{A_{n}(\cdot)}\right)\right\|_{H}^{p}\right) .
$$

The sequence $\left(\left\|\left(\pi_{n}-I_{H}\right)\left(S(t-\cdot, x-*) B\left(X^{-}(\cdot, *)\right) \times 1_{A_{n}(\cdot)}\right)\right\|_{H}\right), n \geqslant 1$, decreases to 0 as $n \rightarrow \infty$. It is bounded by $\sup _{n} 2\left\|S(t-\cdot, x-*) B\left(X^{-}(\cdot, *)\right)\right\|_{H}$; we prove that this last random variable belongs to $L^{p}(\Omega)$. Indeed, The Cauchy-Schwarz inequality implies

$$
\mathrm{E}\left(\sup _{n}\left\|S(t-\cdot, x-*) B\left(X^{-}(\cdot, *)\right)\right\|_{H}^{p}\right) \leqslant C \sum_{i=1}^{4} T_{i}
$$

with 


$$
\begin{aligned}
& T_{1}=\mathrm{E}(\left.\|\left. S(t-\cdot, x-*)\left(1+\mid X^{0}(\cdot, *)\right)||\right|_{H} ^{p}\right), \\
& T_{2}=\mathrm{E}\left(\sup _{n} \mid \int_{0}^{t} \mathrm{~d} s \iint \mathrm{d} y \mathrm{~d} z S(t-s, x-y) f(|y-z|) S(t-s, x-z)\right. \\
& \quad \times\left|\int_{0}^{s_{n}} \int_{\mathbb{R}^{2}} S(s-r, y-\eta)(A+B)(X(r, \eta)) F(\mathrm{~d} r, \mathrm{~d} \eta)\right| \\
&\left.\quad \times\left.\left|\int_{0}^{s_{n}} \int_{\mathbb{R}^{2}} S(s-r, z-\zeta)(A+B)(X(r, \zeta)) F(\mathrm{~d} r, \mathrm{~d} \zeta)\right|\right|^{p / 2}\right), \\
& T_{3}=\mathrm{E}\left(\sup _{n} \mid \int_{0}^{t} \mathrm{~d} s \iint \mathrm{d} y \mathrm{~d} z S(t-s, x-y) f(|y-z|) S(t-s, x-z) .\right. \\
& \quad \times\left|\left\langle 1_{\left[0, s_{n}\right]}(\cdot) S(s-\cdot, y-*) D(X(\cdot, *)), h\right\rangle_{H}\right| \\
&\left.\quad \times\left.\left|\left\langle 1_{\left[0, s_{n}\right]}(\cdot) S(s-\cdot, z-*) D(X(\cdot, *)), h\right\rangle_{H}\right|\right|^{p / 2}\right), \\
& \quad \\
& \quad\left(\sup _{n} \mid \int_{0}^{t} \mathrm{~d} s \iint \mathrm{d} y \mathrm{~d} z S(t-s, x-y) f(|y-z|) S(t-s, x-z)\right. \\
& \quad \times\left|\int_{0}^{s_{n}} \int_{\mathbb{R}^{2}} S(s-r, y-\eta) b(X(r, \eta)) \mathrm{d} r \mathrm{~d} \eta\right| \\
&\left.\quad\left|\int_{0}^{s_{n}} \int_{\mathbb{R}^{2}} S(s-r, z-\zeta) b(X(r, \zeta)) \mathrm{d} r \mathrm{~d} \zeta\right|^{p / 2}\right) .
\end{aligned}
$$

Hölder's inequality implies that

$$
\begin{aligned}
T_{2} \leqslant & C \int_{0}^{t} \mathrm{~d} s \iint \mathrm{d} y \mathrm{~d} z S(t-s, x-y) f(|y-z|) S(t-s, x-z) \\
& \times \mathrm{E}\left(\sup _{n}\left|\int_{0}^{s_{n}} \int_{\mathbb{R}^{2}} S(s-r, y-\eta)(A+B)(X(r, \eta)) F(\mathrm{~d} r, \mathrm{~d} \eta)\right|^{p}\right)^{1 / 2} \\
& \times \mathrm{E}\left(\sup _{n}\left|\int_{0}^{s_{n}} \int_{\mathbb{R}^{2}} S(s-r, z-\zeta)(A+B)(X(r, \zeta)) F(\mathrm{~d} r, \mathrm{~d} \zeta)\right|^{p}\right)^{1 / 2} .
\end{aligned}
$$

Doob's maximal inequality applied to the martingale

$$
\left(\int_{0}^{\tau} \int_{\mathbb{R}^{2}} S(s-r, y-\eta)(A+B)(X(r, \eta)) F(\mathrm{~d} r, \mathrm{~d} \eta), \mathscr{F}_{\tau}\right)
$$

and (A.11) imply that 


$$
T_{2} \leqslant C\left[1+\sup _{(s, y) \in[0, T] \times \mathbb{R}^{2}} \mathrm{E}\left(|X(s, y)|^{p}\right)\right] .
$$

A similar, easier computation using The Cauchy-Schwarz's and Hölder's inequality yields

$$
T_{3}+T_{4} \leqslant C\left[1+\sup _{(s, y) \in[0, T] \times \mathbb{R}^{2}} \mathrm{E}\left(|X(s, y)|^{p}\right)\right] .
$$

Therefore, these estimations and (A.15) imply, for $p \in[1, \infty[$,

$$
\mathrm{E}\left(\sup _{n}\left\|S(t-\cdot, x-*) B\left(X^{-}(\cdot, *)\right)\right\|_{H}^{p}\right)<\infty .
$$

Thus, by dominated convergence, the sequence $\left(Z_{n}(t, x)\right)_{n \geqslant 1}$ decreases to 0 . Moreover, $Z_{n}(t, x)$ is jointly continuous in $(t, x)$; consequently, by Dini's theorem,

$$
\sup _{(t, x) \in[0, T] \times K} Z_{n}(t, x) \downarrow 0 \text { as } n \rightarrow \infty .
$$

Thus,

$$
\sup _{(t, x) \in[0, T] \times K} U_{n}^{6,2}(t, x) \underset{n \rightarrow \infty}{\rightarrow} 0 .
$$

The convergences (2.57) and (2.58) complete the proof of (2.53) for $i=6$, and hence that of the proposition.

Proof of Proposition 2.1. Since equation (2.2) defining the process $\{X(t, x),(t, x) \in$ $\left.[0, T] \times \mathbb{R}^{2}\right\}$ is a particular case of (2.1) which defines $\left\{X_{n}(t, x),(t, x) \in[0, T] \times \mathbb{R}^{2}\right\}$, Propositions 2.2 and 2.3 ensure the validity of conditions (P1) and (P2) of Lemma A.2 for the sequence of processes $Y_{n}(t, x):=X_{n}(t, x)-X(t, x)$ and the sequence of adapted sets $B_{n}(t):=A_{n}(t)$ defined in (2.10). Therefore, given any $0<\gamma<\beta / 2(1+\beta), p \in[1, \infty)$,

$$
\lim _{n \rightarrow \infty} \mathrm{E}\left(\left\|X_{n}-X\right\|_{\gamma, K}^{p} 1_{A_{n}(T)}\right)=0
$$

where $\|\cdot\|_{\gamma, K}$ is given by (1.5).

Fix $\varepsilon>0$; by Lemma 2.1 there exists $n_{0} \in \mathbb{N}$ such that $P\left(A_{n}(T)^{c}\right)<\varepsilon$ for $n \geqslant n_{0}$. Then, for any $\eta>0$,

$$
\begin{aligned}
P\left(\left\|X_{n}-X\right\|_{\gamma, K}>\eta\right) & \leqslant \varepsilon+P\left(\left\|X_{n}-X\right\|_{\gamma, K}>\eta, A_{n}(T)\right) \\
& \leqslant \varepsilon+\eta^{-p} \mathrm{E}\left(\left\|X_{n}-X\right\|_{\gamma, K}^{p} 1_{A_{n}(T)}\right) .
\end{aligned}
$$

Since $\varepsilon>0$ is arbitrary, (2.59) and (2.60) show (2.4).

\section{Approximation in $L^{p}$}

In the previous section, we proved an approximation theorem in probability, by showing the $L^{p}$ convergence of the sequence $X_{n}$ localized by $A_{n, M(n)}$. The aim of this section is to check that under a stronger growth assumption on the coefficients, a slight modification of the proof 
yields the $L^{p}$-convergence of $X^{n}$ to $X$ without localization. Let us introduce the following growth condition:

$\left(\mathrm{C4}^{\prime}\right)$ There exists $\delta \in(0,1)$ and a constant $C>0$ such that, for $x \in \mathbb{R}^{2}$,

$$
|A(x)|+|B(x)|+|D(x)|+|b(x)| \leqslant C\left(1+|x|^{\delta}\right) .
$$

Then we have the following proposition:

Proposition 3.1. Assume (C1), (C2) and $\left(\mathrm{C}^{\prime}\right)$, and let $X$ and $X^{n}$ be defined by (2.1) and (2.2), respectively. For any $\gamma \in(0, \beta / 2(1+\beta))$, every compact subset $K \subset \mathbb{R}^{2}$ and every $p \in[1,+\infty)$,

$$
\lim _{n}\|\| X_{n}-X\left\|_{\gamma, K}\right\|_{p}=0
$$

The proof is very similar to that of Proposition 2.1, and will only be sketched. It depends on several technical lemmas, which are 'unlocalized' versions of Lemmas 2.3 and 2.4.

Lemma 3.1. Suppose that conditions (C1), (C2) and (C4') hold. Then, for any $p \in[1,+\infty)$, $\left.t \in[0, T], \delta^{\prime} \in\right] \delta, 1[$ and $n \geqslant 1$,

$$
\begin{aligned}
& \sup _{(s, y) \in[0, t] \times \mathbb{R}^{2}} \mathrm{E}\left(\left|X_{n}^{k}(s, y)-\bar{X}_{n}^{k}(s, y)\right|^{p}\right) \\
& \leqslant C n^{p / 2} 2^{-n(1+\beta) p / 2}\left[1+\sup _{(s, y) \in[0, t] \times \mathbb{R}^{2}} \mathrm{E}\left(\left|X_{n}^{k-1}(s, y)\right|^{\delta^{\prime} p}\right)\right]
\end{aligned}
$$

and

$$
\sup _{(s, y) \in[0, t] \times \mathbb{R}^{2}} \mathrm{E}\left(\left|X_{n}(s, y)-X_{n}^{-}(s, y)\right|^{p}\right) \leqslant C n^{p / 2} 2^{-n(1+\beta) p / 2}\left[1+\sup _{(s, y) \in[0, t] \times \mathbb{R}^{2}} \mathrm{E}\left(\left|X_{n}(s, y)\right|^{\delta p}\right)\right] .
$$

Proof. Consider the decomposition

$$
\mathrm{E}\left(\left|X_{n}^{k}(t, x)-\bar{X}_{n}^{k}(t, x)\right|^{p}\right) \leqslant C \sum_{i=1}^{4} \tilde{T}^{k, i}
$$

where each term $\tilde{T}_{n}^{k, i}$ is deduced from the corresponding term $T_{n}^{k, i}$ introduced in (2.24) by removing $1_{A_{n}(t)}$.

Let $\bar{p}$ and $\bar{q}$ be conjugate exponents such that $\delta \bar{p}=\delta^{\prime}$. Then The Cauchy-Schwarz and Hölder inequalities, along with (1.13) and (A.11), imply 


$$
\begin{aligned}
\tilde{T}^{k, 2}(t, x) & \leqslant \mathrm{E}\left(\left\|1_{\left(t_{n}, t\right]} \omega_{n}\right\|_{H}^{\bar{q} p}\right)^{1 / \bar{q}} \mathrm{E}\left(\left\|1_{\left[t_{n}, t\right]}(\cdot) S(t-\cdot, x-*) B\left(X_{n}^{k-1}(\cdot, *)\right)\right\|_{H}^{\bar{p} p}\right)^{1 / \bar{p}} \\
& \leqslant C n^{p / 2} 2^{n p / 2} 2^{-n p / 2}\left[\mu\left(t-t_{n}\right)^{p \bar{p} / 2}\left(1+\sup _{(s, y) \in[0, t] \times \mathbb{R}^{2}} \mathrm{E}\left(\left|X_{n}^{k-1}(s, y)\right|^{\delta \bar{p} p}\right)\right)\right]^{1 / \bar{p}} \\
& \leqslant C n^{p / 2} 2^{-n((1+\beta) / 2) p}\left[1+\sup _{(s, y) \in[0, t] \times \mathbb{R}^{2}} \mathrm{E}\left(\left|X_{n}^{k-1}(s, y)\right|^{\delta^{\prime} p}\right)\right] .
\end{aligned}
$$

The upper estimates of $\tilde{T}_{n}^{k, i}, \quad i=1,3,4$ are obtained by means of a straightforward modification of those of $T_{n}^{k, i}$ in the proof of Lemma 2.3; this concludes the proof of (3.2).

Using the arguments in the proof of Millet and Sanz-Solé (1999, Theorem 1.2), we obtain the convergence of the Picard iteration scheme, that is, for $p \in[1,+\infty)$,

$$
\lim _{k \rightarrow \infty} \sup _{(s, x) \in[0, T] \times \mathbb{R}^{2}}\left(\left\|X_{n}^{k}(s, x)-X_{n}(s, x)\right\|_{p}+\left\|\bar{X}_{n}^{k}(s, x)-X_{n}^{-}(s, x)\right\|_{p}\right)=0 .
$$

Therefore, (3.2) and (3.4) imply (3.3).

We now prove $L^{p}$-convergence of $X_{n}^{-}(s, y)$ to $X_{n}(s, y)$.

Lemma 3.2. Assume (C1), (C2) and (C4'); then, for $p \in[1,+\infty)$,

$$
\sup _{n \geqslant 1} \sup _{(t, x) \in[0, T] \times \mathbb{R}^{2}}\left(\left\|X_{n}(t, x)\right\|_{p}+\left\|X_{n}^{-}(t, x)\right\|_{p}\right)<\infty
$$

and

$$
\sup _{(t, x) \in[0, T] \times \mathbb{R}^{2}}\left\|X_{n}(t, x)-X_{n}^{-}(t, x)\right\|_{p} \leqslant C n^{1 / 2} 2^{-n(1+\beta) / 2} .
$$

Proof. The proof reduces to that of

$$
\sup _{n \geqslant 1} \sup _{k \geqslant 0} \sup _{(t, x) \in[0, T] \times \mathbb{R}^{2}}\left(\left\|X_{n}^{k}(t, x)\right\|_{p}+\left\|\bar{X}_{n}^{k}(t, x)\right\|_{p}\right)<\infty .
$$

Indeed, (3.4) and (3.7) imply (3.5), while (3.3) and (3.5) yield (3.6). For $r \leqslant t$, consider the decomposition

$$
\mathrm{E}\left(\left|X_{n}^{k+1}(t, r ; x)\right|^{p}\right) \leqslant C \sum_{i=1}^{6} \tilde{T}_{n}^{k+1, i}(t, r ; x),
$$

where $\tilde{T}_{n}^{k+1, i}(t, r ; x)$ is deduced from the term $T_{n}^{k+1, i}(t, r ; x)$ in (2.33) by removing $1_{A_{n}(t)}$. The arguments used to obtain upper estimates of $\tilde{T}_{n}^{k+1, i}$ for $i \neq 4$ are similar to that in Lemma 2.4 and are omitted. They yield the analogues of (2.34)-(2.36), (2.38) and (2.39).

Let $\left.\delta^{\prime} \in\right] \delta, 1\left[, \bar{p}=\left(\delta^{\prime}\right)^{-1}, \bar{p}\right.$ and $\bar{q}$ be conjugate exponents; then The Cauchy-Schwarz and Hölder inequalities, along with (A.11) and (1.12), yield 


$$
\begin{aligned}
\tilde{T}_{n}^{k+1,4}(t, r ; x) & \leqslant \mathrm{E}\left(\left\|\omega^{n}\right\|_{H}^{\bar{q} p}\right)^{1 / \bar{q}} \mathrm{E}\left(\left\|S(t-\cdot, x-*) 1_{[0, r]}(\cdot)\left[B\left(X_{n}^{k}\right)-B\left(\bar{X}_{n}^{k}\right)\right](\cdot, *)\right\|_{H}^{\bar{p} p}\right)^{1 / \bar{p}} \\
& \leqslant C n^{p / 2} 2^{\mathrm{n} p / 2}\left[\sup _{(s, y) \in[0, r] \times \mathbb{R}^{2}} \mathrm{E}\left(\left|\left(X_{n}^{k}-\bar{X}_{n}^{k}\right)(s, y)\right|^{\bar{p} p}\right)\right]^{1 / \bar{p}} .
\end{aligned}
$$

Hence (3.2) implies

$$
\begin{aligned}
\tilde{T}_{n}^{k+1,4}(t, r ; x) & \leqslant C n^{p / 2} 2^{n p / 2}\left\{n^{\bar{p} p / 2} 2^{-n(1+\beta) \bar{p} p / 2}\left[1+\sup _{(s, y) \in[0, r] \times \mathbb{R}^{2}} \mathrm{E}\left(\left|X_{n}^{k-1}(s, y)\right|^{\delta^{\prime} \bar{p} p}\right)\right]\right\}^{1 / \bar{p}} \\
& \leqslant C n^{p} 2^{-n \beta p / 2}\left[1+\sup _{(s, y) \in[0, r] \times \mathbb{R}^{2}} \mathrm{E}\left(\left|X_{n}^{k-1}(s, y)\right|^{p}\right)\right] .
\end{aligned}
$$

Set $\tilde{\varphi}_{n}^{-1} \equiv 0$ and, for every $k \geqslant 0$,

$$
\tilde{\varphi}_{n}^{k}(t)=\sup _{0 \leqslant s \leqslant t} \sup _{y \in \mathbb{R}^{2}} \mathrm{E}\left(\left|X_{n}^{k}(s, y)\right|^{p}+\left|\bar{X}_{n}^{k}(s, y)\right|^{p}\right) .
$$

Then, for every $k \geqslant 0$,

$$
\tilde{\varphi}_{n}^{k+1}(t) \leqslant C \int_{0}^{t}\left[1+\tilde{\varphi}_{n}^{k}(s)+\tilde{\varphi}_{n}^{k-1}(s)\right] \mathrm{d} s .
$$

Since $\sup _{0 \leqslant t \leqslant T} \tilde{\varphi}_{n}^{0}(t)=C<\infty$, this implies (3.7).

Replacing (2.30) and (2.31) by (3.5) and (3.6) respectively, the arguments in the proofs of Propositions 2.2 and 2.3 yield the following result:

Proposition 3.2. Assume (C1), (C2) and (C4'). Let $K$ be a compact subset of $\mathbb{R}^{2}$ and $p \in[1, \infty)$; then we have the following:

(i) For $0<\gamma<\beta /(2(1+\beta)), 0 \leqslant t \leqslant \bar{t} \leqslant T, x, \bar{x} \in K$,

$$
\sup _{n}\left\|X_{n}(t, x)-X_{n}(\bar{t}, \bar{x})\right\|_{p}+\|X(t, x)-X(\bar{t}, \bar{x})\|_{p} \leqslant C\left(|t-\bar{t}|^{\gamma}+|x-\bar{x}|^{\gamma}\right) .
$$

(ii) $\operatorname{For}(t, x) \in[0, T] x K$,

$$
\lim _{n}\left\|X_{n}(t, x)-X(t, x)\right\|_{p}=0 .
$$

Proof of Proposition 3.1. To conclude the proof of this proposition, it suffices to apply Bally et al. (1995, Lemma A.1). Indeed, the results proved in the previous proposition ensure the validity of the hypothesis of that lemma.

\section{Appendix}

This section quotes some notation introduced in our previous paper (Millet and Sanz-Solé 1999), which is extensively used along the paper. It also contains some technical results. 
For any $t \in[0, T], h \geqslant 0, \xi \in \mathbb{R}^{2}$, set

$$
\begin{aligned}
& J(t)=\int_{|y|<|x|<t} \frac{1}{\sqrt{t^{2}-|x|^{2}}} f(|x-y|) \frac{1}{\sqrt{t^{2}-|y|^{2}}} \mathrm{~d} x \mathrm{~d} y, \\
& \mu(t)=\int_{0}^{t} \mathrm{~d} s \int_{\mathbb{R}^{2}} \mathrm{~d} x \int_{\mathbb{R}^{2}} \mathrm{~d} y S(s, x) f(|x-y|) S(s, y)=\frac{1}{2 \pi^{2}} \int_{0}^{t} J(s) \mathrm{d} s, \\
& v(t)=\frac{1}{2 \pi} \int_{0}^{t} \mathrm{~d} s \int_{|x|<s} \frac{\mathrm{d} x}{\sqrt{s^{2}-|x|^{2}}}=\frac{t^{2}}{2}, \\
& v_{t, h}=\int_{0}^{t} \mathrm{~d} s \int_{|y|<s} \mathrm{~d} y(S(s, y)-S(s+h, y)), \\
& \tilde{v}_{t, h}=\int_{0}^{t} \mathrm{~d} s \int_{s \leqslant|y|<s+h} \mathrm{~d} y S(s+h, y), \\
& \mu_{t, h}=\int_{0}^{t} \mathrm{~d} s \int_{|y|<s} \mathrm{~d} y \int_{|z|<s} \mathrm{~d} z[S(s, y)-S(s+h, y)] f(|y-z|)[S(s, z)-S(s+h, z)], \\
& \tilde{\mu}_{t, h}=\int_{0}^{t} \mathrm{~d} s \int_{s \leqslant|y|<s+h} \mathrm{~d} y \int_{s \leqslant|z|<s+h} \mathrm{~d} z S(s+h, y) f(|y-z|) S(s+h, z), \\
& M_{t, \xi}=\int_{0}^{t} \int_{\substack{|y|<s \\
|y-\xi| \geqslant s}} \mathrm{~d} y \int_{\substack{|z|<s \\
|z-\xi| \geqslant s}} \mathrm{~d} z S(s, y) f(|y-z|) S(s, z), \\
& N_{t, \xi}=\int_{\frac{|\xi|}{2}}^{t} \mathrm{~d} s \int_{\substack{|y|<s \\
|y-\xi|<s}} \mathrm{~d} y \int_{\substack{|z|<s \\
|z-\xi|<s}} \mathrm{~d} z|S(s, y)-S(s, y-\xi)| f(|y-z|)|S(s, z)-S(s, z-\xi)| .
\end{aligned}
$$

A direct computation shows that

$$
v_{t, h}+\tilde{v}_{t, h} \leqslant C h^{1 / 2} .
$$

Assume that $f$ satisfies assumption (C1); then Lemma A.1 in Millet and Sanz-Solé (1999) implies that

$$
J(t) \leqslant C t^{\beta}, \quad \mu(t) \leqslant C t^{\beta+1}, \quad t \in[0, T],
$$

while, for $t \in[0, T], h \vee|\xi| \leqslant \frac{1}{2}$ and $0<\delta<\beta /(1+\beta)$, Lemma A.5 in Millet and Sanz-Solé (1999) shows that

$$
\begin{gathered}
\mu_{t, h}+\tilde{\mu}_{t, h} \leqslant C h^{\delta}, \\
M_{t, \xi}+N_{t, \xi} \leqslant C|\xi|^{\delta} .
\end{gathered}
$$

Furthermore, (A.24) in Millet and Sanz-Solé (1999) yields that for $0 \leqslant s \leqslant T, x \in \mathbb{R}^{2}$, 


$$
\int_{|x| / 2}^{x} \mathrm{~d} r \int_{|z-x|<|z|<s} \mathrm{~d} z(S(r, z)-S(r, z-x)) \leqslant C|x|^{1 / 2} .
$$

The following result is a slight generalization of Theorem 1.2 in Millet and Sanz-Solé (1999).

Lemma A.1. Suppose (C1), (C2) end (C'3); for any $T>0$, equation (2.1) (equation (2.2)) has a unique solution $X_{n}(X)$ on $[0, T] \times \mathbb{R}^{2}$. Futhermore,

$$
\sup _{0 \leqslant t \leqslant T} \sup _{x \in \mathbb{R}^{2}} \mathrm{E}\left(|X(t, x)|^{p}\right)<\infty, \quad p \in[1, \infty) .
$$

Sketch of proof. Since (2.2) is a particular case of (2.1), we only prove the existence of the solution to (2.1). The argument for (2.2) is simpler, since no localization is required. Given $M>0$, consider the evolution equation

$$
\begin{aligned}
X_{n, M}(t, x)= & X^{0}(t, x)+\int_{0}^{t} \int_{\mathbb{R}^{2}} S(t-s, x-y) A\left(X_{n, M}(s, y)\right) F(\mathrm{~d} s, \mathrm{~d} y) \\
& +\left\langle S(t-\cdot, x-*) B\left(X_{n, M}(\cdot, *)\right), \omega^{n, M}\right\rangle_{H} \\
& +\left\langle S(t-\cdot, x-*) D\left(X_{n, M}(\cdot, *)\right), h\right\rangle_{H} \\
& +\int_{0}^{t} \int_{\mathbb{R}^{2}} S(t-s, x-y) b\left(X_{n, M}(s, y)\right) \mathrm{d} s \mathrm{~d} y .
\end{aligned}
$$

A slight extension of the proof of Millet and Sanz-Solé (1999, Theorem 1.2) based on Picard's iteration scheme - using The Cauchy-Schwarz's inequality for the scalar products, Gronwall's lemma and (A.11) - provides the existence and uniqueness of the solution to (A.16). For any $(t, x) \in[0,1] \times \mathbb{R}^{2}$, define

$$
X_{n}(t, x)=X_{n, M}(t, x) \quad \text { on } A_{n, M}(T) .
$$

For fixed $n$ and $M$, the sets $\left(A_{n, M}(t)\right)_{t \in[0, T]}$ are decreasing in $t$. Therefore, a standard argument based on the local property of stochastic integrals implies that this definition is consistent and, since $P\left(\cup_{M \geqslant 1} A_{n, M}(1)\right)=1$ for every integer $n$, this provides the existence and uniqueness of solution to equation (2.1).

The proof of (A.15) is a straightforward extension of the corresponding inequality (1.8) in Millet and Sanz-Solé (1999).

The following lemma is a localized version of Lemma A.1 in Bally et al. (1995). For the sake of completeness we give the main arguments of the proof.

Lemma A.2. Let $\left\{Y_{n}(t, x),(t, x) \in K_{0}\right\}, n \geqslant 1$, be a sequence of processes indexed by $K_{0}=[0, T] \times K, K$ being a compact set of $\mathbb{R}^{2}$. Let $\left\{B_{n}(t), t \in[0, T]\right\} \subset \mathscr{F}$ be a sequence of adapted sets which, for every $n$, decreases in t. Make the following assumptions, for every $p \in(1, \infty)$ : 
(P1) There exists $\delta>0$ such that, for any $0 \leqslant t \leqslant \bar{t} \leqslant T, x, \bar{x} \in K$,

$$
\sup _{n} \mathrm{E}\left(\left|Y_{n}(t, x)-Y_{n}(\bar{t}, \bar{x})\right|^{p} 1_{B_{n}(\bar{t})}\right) \leqslant C(|t-\bar{t}|+|x-\bar{x}|)^{3+\delta} .
$$

(P2) For every $(t, x) \in[0, T] \times K$,

$$
\lim _{n \rightarrow \infty} \mathrm{E}\left(\left|Y_{n}(t, x)\right|^{p} 1_{B_{n}(t)}\right)=0 .
$$

Then, for any $\rho \in(0, \delta / p)$ and any $r \in[1, p)$,

$$
\lim _{n \rightarrow \infty} \mathrm{E}\left(\left\|Y_{n}\right\|_{\rho, K}^{r} 1_{B_{n}(T)}\right)=0 .
$$

Proof. Let $\zeta=2 d+\delta^{\prime}, d=3,0<\delta^{\prime}<\delta$; set $z=(t, x), \bar{z}=(\bar{t}, \bar{x})$. Then, by (P1),

$$
\int_{K_{0}} \int_{K_{0}} \mathrm{E}\left(\frac{\left|Y_{n}(z)-Y_{n}(\bar{z})\right|^{p}}{|z-\bar{z}|^{\zeta}} 1_{B_{n}(\bar{t})}\right) \mathrm{d} z \mathrm{~d} \bar{z} \leqslant C B^{\prime}
$$

where

$$
B^{\prime}=\int_{K_{0}} \int_{K_{0}}|z-\bar{z}|^{-d+\delta-\delta^{\prime}} \mathrm{d} z \mathrm{~d} \bar{z}<+\infty .
$$

Set

$$
Z=\int_{K_{0}} \int_{K_{0}} \frac{\left|Y_{n}(z)-Y_{n}(\bar{z})\right|^{p}}{|z-\bar{z}|^{\xi}} 1_{B_{n}(\bar{t})} \mathrm{d} z \mathrm{~d} \bar{z} .
$$

Clearly, by Fubini's theorem, $\mathrm{E}(Z) \leqslant C B^{\prime}$, so that

$$
\mathrm{P}\left(Z>\lambda^{p}\right) \leqslant C \lambda^{-p} B^{\prime} .
$$

The Garsia-Rodemich-Rumsey lemma yields

$$
\left|Y_{n}(z)-Y_{n}(\bar{z})\right| 1_{B_{n}(\bar{t})} \leqslant \bar{C} Z^{1 / p}|z-\bar{z}|^{\rho_{0}},
$$

with $\rho_{0}=\delta^{\prime} / p$. Since $\left\{B_{n}(t), t \in[0, T]\right\}$ decreases in $t$, this yields, for any $\rho<\delta / p$,

$$
\begin{aligned}
P\left(\sup _{z \neq \bar{z}} \frac{\left|Y_{n}(z)-Y_{n}(\bar{z})\right|}{|z-\bar{z}|^{\rho}}>\lambda, B_{n}(T)\right) & \leqslant \lambda^{-p} \mathrm{E}\left(\sup _{z \neq \bar{z}} \frac{\left|Y_{n}(z)-Y_{n}(\bar{z})\right|^{p}}{|z-\bar{z}|^{\rho p}} 1_{B_{n}(T)}\right) \\
& \leqslant C \lambda^{-p} \mathrm{E}(Z) \leqslant C \lambda^{-p} .
\end{aligned}
$$

On the set $B_{n}(T)$, we now proceed exactly as in Bally et al. (1995, Lemma A.1) and show that for any $\varepsilon>0, r \in[1, p)$, there exists $N \in \mathbb{N}$ such that, for any $n \geqslant N$,

$$
\mathrm{E}\left(\left\|Y_{n}\right\|_{\beta, K}^{r} 1_{B_{n}(T)}\right) \leqslant \varepsilon^{r}+C \varepsilon
$$

Remark A.1. Under condition (C1), inequalities (A.12) and (A.13) have been improved in a recent paper of Millet and Morien (2000) as follows. For $0<\delta<\beta<1$, 


$$
\mu_{t, h}+\tilde{\mu}_{t, h} \leqslant C h^{\delta}, \quad M_{t, \xi}+N_{t, \xi} \leqslant C|\xi|^{\delta} .
$$

Suppose that $(\mathrm{C} 2)$ is replaced by the following condition:

$\left(\mathrm{C} 2^{\prime}\right) u_{0}: \mathbb{R}^{2} \rightarrow \mathbb{R}^{2}$ is of class $\mathscr{C}^{1}$ and bounded with $\beta$-Hölder continuous partial derivatives, and $v_{0}: \mathbb{R}^{2} \rightarrow \mathbb{R}^{2}$ is such that $\left|v_{0}\right|+\left|\nabla v_{0}\right| \in L^{q_{0}}$ for some $\left.q_{0} \in\right] 4 \vee 2 /(1-\beta)[$.

The processes $u$ and $X$ have almost surely $\gamma$-Hölder continuous trajectories for $0<\gamma<\beta$, and the same arguments show that Theorem 2.1 can be reinforced to characterize the topological support of $u$ in $\mathscr{C}^{\gamma}([0, T] \times K)$ for $\left.\gamma \in\right] 0, \beta[$. All intermediary results can also be stated for $\gamma \in] 0, \beta[$ instead of $\gamma \in] 0, \beta /(2(1+\beta))[$.

\section{Acknowledgements}

Part of this work was done during a visit of the authors to the Mathematical Sciences Research Institute at Berkeley. We would like to thank the hospitality and support provided by them. Both authors have also been partially supported by grant ERBF MRX CT960075A from the European Union. The second named author has been supported by grant PB96-0088 from the Subdirección General de Formación y Promoción del Conocimiento.

\section{References}

Bally, V., Millet, A. and Sanz-Solé, M. (1995) Approximation and support theorem in Hölder norm for parabolic stochastic partial differential equations. Ann. Probab., 23, 178-222.

Dalang, R. and Frangos, N. (1998) The stochastic wave equation in two spatial dimensions. Annals of Probab., 26, 187-212.

Millet, A. and Sanz-Solé, M. (1994a) The support of an hyperbolic stochastic partial differential equation. Probab. Theory Related Fields, 98, 361-387.

Millet, A. and Sanz-Solé, M. (1994b) A simple proof of the support theorem for diffusion processes. In J. Azéma, P.-A. Meyer and M. Yor (eds), Séminaire de Probabilités XXVIII, Lecture Notes in Math. 1583, pp. 36-48. Berlin: Springer-Verlag.

Millet, A. and Sanz-Solé, M. (1999) A stochastic wave equation in two space dimensions: Smoothness of the law. Ann. Probab., 27, 803-844.

Millet, A. and Morien, P.-L. (2000) On a stochastic wave equation in two space dimensions: regularity of the solution and its density. Stoch. Proc. Appl., 86, 141-162.

Received April 1988 and revised November 1999 